ISSN 1855-3966 (printed edn.), ISSN 1855-3974 (electronic edn.)

\author{
ARS MATHEMATICA CONTEMPORANEA 15 (2018) 297-321 \\ https://doi.org/10.26493/1855-3974.806.c9d \\ (Also available at http://amc-journal.eu)
}

\title{
Finite actions on the 2-sphere, the projective plane and I-bundles over the projective plane
}

\author{
John Kalliongis \\ Department of Mathematics and Statistics, Saint Louis University, \\ 220 North Grand Boulevard, Saint Louis, MO 63103 \\ Ryo Ohashi \\ Department of Mathematics and Computer Science, King's College, \\ 133 North River Street, Wilkes-Barre, PA 18711
}

Received 5 February 2015, accepted 6 February 2018, published online 25 June 2018

\begin{abstract}
In this paper, we consider the finite groups which act on the 2-sphere $\mathbb{S}^{2}$ and the projective plane $\mathbb{P}^{2}$, and show how to visualize these actions which are explicitly defined. We obtain their quotient types by distinguishing a fundamental domain for each action and identifying its boundary. If $G$ is an action on $\mathbb{P}^{2}$, then $G$ is isomorphic to one of the following groups: $\mathbb{S}_{4}, \mathbb{A}_{5}, \mathbb{A}_{4}, \mathbb{Z}_{m}$ or $\operatorname{Dih}\left(\mathbb{Z}_{m}\right)$. For each group, there is only one equivalence class (conjugation), and $G$ leaves an orientation reversing loop invariant if and only if $G$ is isomorphic to either $\mathbb{Z}_{m}$ or $\operatorname{Dih}\left(\mathbb{Z}_{m}\right)$. Using these preliminary results, we classify and enumerate the finite groups, up to equivalence, which act on $\mathbb{P}^{2} \times I$ and the twisted I-bundle over $\mathbb{P}^{2}$. As an example, if $m>2$ is an even integer and $m / 2$ is odd, there are three equivalence classes of orientation reversing $\operatorname{Dih}\left(\mathbb{Z}_{m}\right)$-actions on the twisted I-bundle over $\mathbb{P}^{2}$. However if $m / 2$ is even, then there are two equivalence classes.
\end{abstract}

Keywords: Achiral symmetry, chiral symmetry, equivalence of actions, finite group action, isometry, orbifold, symmetry.

Math. Subj. Class.: 57S25, 05E18, 57M60, 57R18, 58D19, 57M20

\section{Introduction}

The finite orientation preserving groups which act effectively on $\mathbb{S}^{2}$ are known. (See for example Gross and Tucker [5] and Zimmermann [9].) They are the octahedral symmetric group $\mathbb{S}_{4}$, the dodecahedral/icosahedral alternating group $\mathbb{A}_{5}$, the tetrahedral alternating

E-mail addresses: kalliongisje@slu.edu (John Kalliongis), ryoohashi@kings.edu (Ryo Ohashi) 
group $\mathbb{A}_{4}$, the cyclic group $\mathbb{Z}_{m}$ or the dihedral group $\operatorname{Dih}\left(\mathbb{Z}_{m}\right)$. Using this classification, the actions on the projective plane $\mathbb{P}^{2}$ are also known as folklore, and one can easily compute them by this theorem of Singerman [7] and Tucker [8].

Theorem. Let $F$ be a closed non-orientable surface and let $p: \tilde{F} \rightarrow F$ be the orientable double cover with covering translation $t: \tilde{F} \rightarrow \tilde{F}$. Then any finite group $G$ acting on $F$, lifts to an orientation preserving action of $G$ on $\tilde{F}$ that commutes with $t$. Moreover, the action of $G$ on $F$ is determined by the action of $G \times\langle t\rangle$ on $\tilde{F}$.

If $t: \mathbb{S}^{2} \rightarrow \mathbb{S}^{2}$ is the covering translation such that $\mathbb{S}^{2} /\langle t\rangle=\mathbb{P}^{2}$, one checks that any rotation of $\mathbb{S}^{2}$ commutes with $t$. Therefore since these groups consists of rotations, it follows that the orientation preserving actions on $\mathbb{S}^{2}$ project to $\mathbb{P}^{2}$, giving the following corollary.

Corollary. Any finite group acting on $\mathbb{P}^{2}$ is isomorphic to one of the following groups: $\mathbb{S}_{4}$, $\mathbb{A}_{5}, \mathbb{A}_{4}, \mathbb{Z}_{m}$ or $\operatorname{Dih}\left(\mathbb{Z}_{m}\right)$.

A finite $G$-action on a manifold $M$ is a monomorphism $\varphi: G \rightarrow \operatorname{Homeo}(M)$, where $G$ is a finite group, and $\operatorname{Homeo}(M)$ is the group of homeomorphisms of $M$. Two actions $\varphi_{1}$ and $\varphi_{2}$ are equivalent if there exists a homeomorphism $h$ of $M$ such that $h \varphi_{1}(G) h^{-1}=$ $\varphi_{2}(G)$. For an action $\varphi$, the quotient space $M / \varphi$ is an orbifold which is referred to as the quotient type of the action.

In this paper, we describe how to visualize the finite groups which act on the 2-sphere $\mathbb{S}^{2}$ and the projective plane $\mathbb{P}^{2}$, and show how to obtain their quotient types. Our approach, for the groups which are not cyclic or dihedral, is to view these groups as subgroups of the symmetric group $S_{n}$ for an appropriate $n$, tiling the 2 -sphere with appropriate polygons with $n$ vertices for each group, and explicitly defining each action. As for the cyclic and dihedral groups, we use spherical coordinates to precisely describe their actions on $\mathbb{S}^{2}$. For all these groups, we can easily identify an explicit fundamental region for each action and see its quotient type, which is obtained by identifying the boundary of the fundamental region. In this way, it is easy to see the actions on $\mathbb{S}^{2}, \mathbb{P}^{2}$ and their quotient types. This part of the paper may be considered expository, and we obtain the following theorem where the description of these quotient types may be found in Figure 1.

Theorem 7.1. Let $\varphi: G \rightarrow \operatorname{Homeo}\left(\mathbb{P}^{2}\right)$ be a finite group action on $\mathbb{P}^{2}$. Then $G$ is isomorphic to one of the following groups: $\mathbb{S}_{4}, \mathbb{A}_{5}, \mathbb{A}_{4}, \mathbb{Z}_{m}$ or $\operatorname{Dih}\left(\mathbb{Z}_{m}\right)$. The orbifold quotient $\mathbb{P}^{2} / \varphi$ is an orbifold homeomorphic to one of the following orbifolds: $O^{h}, I^{h}, T^{v}, Z_{m}^{h}$, $S^{2 m}, D_{m}^{v}$ or $D_{m}^{h}$. There is only one equivalence class for each group.

(1) $G \simeq \mathbb{S}_{4}$ if and only if $\mathbb{P}^{2} / \varphi=O^{h}$.

(2) $G \simeq \mathbb{A}_{5}$ if and only if $\mathbb{P}^{2} / \varphi=I^{h}$.

(3) $G \simeq \mathbb{A}_{4}$ if and only if $\mathbb{P}^{2} / \varphi=T^{v}$.

(4) $G \simeq \mathbb{Z}_{m}$ and $m$ is even if and only if $\mathbb{P}^{2} / \varphi=Z_{m}^{h}$.

(5) $G \simeq \mathbb{Z}_{m}$ and $m$ is odd if and only if $\mathbb{P}^{2} / \varphi=S^{2 m}$.

(6) $G \simeq \operatorname{Dih}\left(\mathbb{Z}_{m}\right)$ and $m$ odd if and only if $\mathbb{P}^{2} / \varphi=D_{m}^{v}$.

(7) $G \simeq \operatorname{Dih}\left(\mathbb{Z}_{m}\right)$ and $m$ even if and only if $\mathbb{P}^{2} / \varphi=D_{m}^{h}$. 
This approach relates to topics in topological graph theory found in Gross and Tucker [5]. There, graphs are embedded on surfaces and finite groups act on these spaces with quotient spaces, branch covering maps and branch points, relating to orbifold covering maps and cone points.

Using the above result, we classify in Theorem 7.4 the finite group actions, up to equivalence, on $\mathbb{P}^{2} \times I$ for $I=[0,1]$. If $G$ is an action on $\mathbb{P}^{2} \times I$, then $G$ is isomorphic to one of the following groups: $\mathbb{S}_{4}, \mathbb{S}_{4} \times \mathbb{Z}_{2}, \mathbb{A}_{5}, \mathbb{A}_{5} \times \mathbb{Z}_{2}, \mathbb{A}_{4}, \mathbb{A}_{4} \times \mathbb{Z}_{2}, \mathbb{Z}_{m}, \mathbb{Z}_{m} \times \mathbb{Z}_{2}$, $\operatorname{Dih}\left(\mathbb{Z}_{m}\right)$ or $\operatorname{Dih}\left(\mathbb{Z}_{m}\right) \times \mathbb{Z}_{2}$. We indicate the number of equivalence classes for each group in Theorem 7.4. If $W$ is the twisted I-bundle over the projective plane $\mathbb{P}^{2}$, then we obtain the following results:

Corollary 8.12. Let $\varphi: G \rightarrow \operatorname{Homeo}(W)$ be a finite orientation preserving $G$-action on $W$. Then $G$ is isomorphic to one of the following groups: $\mathbb{S}_{4}, \mathbb{A}_{5}, \mathbb{A}_{4}, \mathbb{Z}_{m}$ or $\operatorname{Dih}\left(\mathbb{Z}_{m}\right)$. The orbifold quotient for each action is a twisted I-bundle orbifold over the following 2-orbifolds: $O^{h}$ (for $\mathbb{S}_{4}$ ), $I^{h}$ (for $\mathbb{A}_{5}$ ), $T^{v}$ (for $\left.\mathbb{A}_{4}\right), Z_{m}^{h}$ (for $\mathbb{Z}_{m}$ and $m$ even), $S^{2 m}$ (for $\mathbb{Z}_{m}$ and $m$ odd $), D_{m}^{v}\left(\right.$ for $\operatorname{Dih}\left(\mathbb{Z}_{m}\right)$ and $m$ odd $)$ and $D_{m}^{h}\left(\right.$ for $\operatorname{Dih}\left(\mathbb{Z}_{m}\right)$ and $m$ even $)$. There is one equivalence class for each quotient type.

Theorem 9.4. Let $\varphi: G \rightarrow \operatorname{Homeo}(W)$ be an orientation reversing $G$-action. Then $G$ is isomorphic to one of the following groups: $\mathbb{S}_{4}, \mathbb{Z}_{m}$ with $m$ even, $\operatorname{Dih}\left(\mathbb{Z}_{m}\right), \mathbb{S}_{4} \times \mathbb{Z}_{2}$, $\mathbb{A}_{5} \times \mathbb{Z}_{2}, \mathbb{A}_{4} \times \mathbb{Z}_{2}, \mathbb{Z}_{m} \times \mathbb{Z}_{2}$ or $\operatorname{Dih}\left(\mathbb{Z}_{m}\right) \times \mathbb{Z}_{2}$

(1) If $G$ is either $\mathbb{S}_{4}, \mathbb{S}_{4} \times \mathbb{Z}_{2}, \mathbb{A}_{5} \times \mathbb{Z}_{2}, \mathbb{A}_{4} \times \mathbb{Z}_{2}, \mathbb{Z}_{m} \times \mathbb{Z}_{2}$ with $m$ even or $\operatorname{Dih}\left(\mathbb{Z}_{m}\right)$ with $m$ odd, there is only one equivalence class.

(2) If $G$ is $\mathbb{Z}_{m}$ with $m>2$ even and $m / 2$ odd, then there are two equivalence classes of $\mathbb{Z}_{m}=\mathbb{Z}_{m / 2} \times \mathbb{Z}_{2}$-actions on $W$.

(3) If $G$ is $\mathbb{Z}_{m}$ with either $m / 2$ even or $m=2$, then there is only one equivalence class.

(4) If $G$ is $\operatorname{Dih}\left(\mathbb{Z}_{m}\right)$ with $m>2$ and $m / 2$ even, there are two equivalence classes of $\operatorname{Dih}\left(\mathbb{Z}_{m}\right)$-actions on $W$.

(5) If $G$ is $\operatorname{Dih}\left(\mathbb{Z}_{m}\right)$ with $m>2$ and $m / 2$ odd, there are three equivalence classes of $\operatorname{Dih}\left(\mathbb{Z}_{m}\right)$-actions on $W$.

(6) If $G$ is $\operatorname{Dih}\left(\mathbb{Z}_{m}\right) \times \mathbb{Z}_{2}$ with $m$ even, there is only one equivalence class.

(7) If $G$ is $\operatorname{Dih}\left(\mathbb{Z}_{m}\right) \times \mathbb{Z}_{2}$ with $m$ odd, then $\operatorname{Dih}\left(\mathbb{Z}_{m}\right) \times \mathbb{Z}_{2} \simeq \operatorname{Dih}\left(\mathbb{Z}_{2 m}\right)$ and there are three equivalence classes of $\operatorname{Dih}\left(\mathbb{Z}_{2 m}\right)$-actions on $W$.

We list all the closed 2-orbifolds with positive Euler number, of which there are 14. (See Figure 1.) In referring to these orbifolds, we use Schönflies notation found in Coxeter and Moser [1], and Dunbar [3].

There are five orientable 2-orbifolds with positive Euler number which have as their underlying space a 2 -sphere with the cone points indicated in the notation. They are $\Sigma(2,2, n)=D_{n}, \Sigma(2,3,3)=T, \Sigma(2,3,4)=O, \Sigma(2,3,5)=I$, and $\Sigma(n, l)=C_{n, l}$. These double cover the following nine non-orientable 2-orbifolds where the double lines are reflector lines. The superscripts $h$ and $v$ stand for horizontal and vertical reflections in their orientable double covers. Except for $\Sigma(n, l)=C_{n, l}$ where the cone points are at the north and south poles, all the cone points are located on the equator.

In this article, where appropriate and depending on the context, we use the same symbol to denote the quotient space and the group acting on $\mathbb{S}^{2}$. For example, $O=\Sigma(2,3,4)$ and $O$ also denote the octahedral group. 

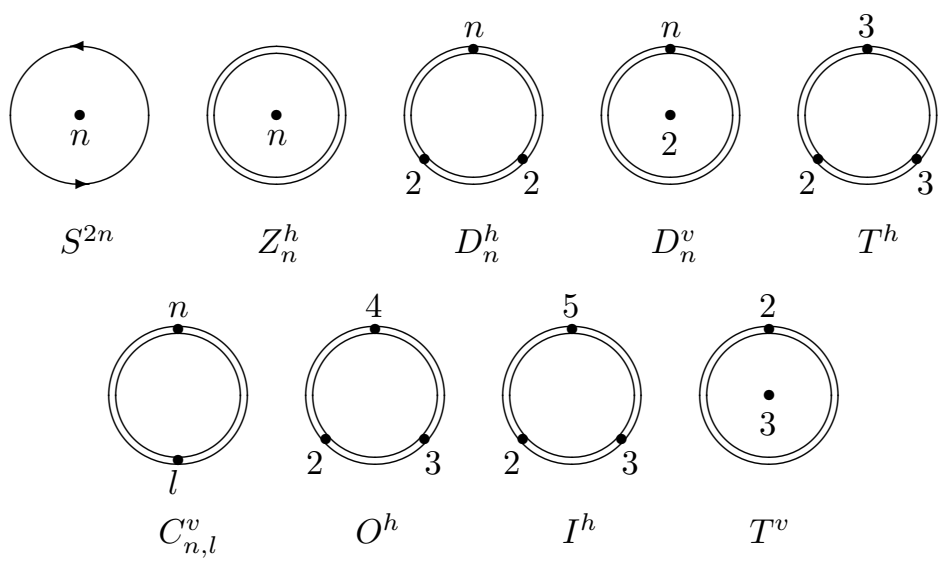

Figure 1: The nine non-orientable 2-orbifolds of positive Euler number.

Here is a brief outline of the paper. We consider each of these orbifolds in Sections 2 through 6, and give model maps which we consider as standard actions, to obtain each quotient type. Summarizing we give the main results for finite actions on $\mathbb{P}^{2}$ and $\mathbb{P}^{2} \times I$ in Section 7. Sections 8 and 9 are devoted to classifying the finite actions on the twisted I-bundle $W$ over $\mathbb{P}^{2}$.

The authors wish to thank the referees for many helpful comments and suggestions.

\section{Chiral octahedral symmetry $O$ and achiral octahedral symmetry $O^{h}$}

We describe the groups $O=\mathbb{S}_{4}$ and $O^{h}=\mathbb{S}_{4} \times \mathbb{Z}_{2}$ acting on the 2-sphere $\mathbb{S}^{2}$, and show how $O$ acts on the projective plane $\mathbb{P}^{2}$. We view $\mathbb{S}^{2}$ as an octahedron which has eight triangles (faces): $\triangle 125, \triangle 145, \triangle 126, \triangle 146, \triangle 235, \triangle 236, \triangle 345$ and $\triangle 346$. (See Figure 2.)

Consider elements of $\mathbb{S}_{6}$ where $a=(1,2)(3,4)(5,6)$ and $b=(1,2,5)(3,4,6)$. The two elements act on the octahedron. We can see that $a$ is a $180^{\circ}$ rotation about the axis passing through the midpoint of edges $\overline{1,2}$ and $\overline{3,4}$. On the other hand, $b$ is a $120^{\circ}$ rotation about the axis passing through the barycenter of $\triangle 125$ and $\triangle 346$ respectively. Further, $a b=(2,6,4,5)$ where $a b$ is a $90^{\circ}$ rotation about the axis passing through vertices 1 and 3 . As a result, the two elements $a$ and $b$ generate a group isomorphic to $\mathbb{S}_{4}$, and we denote this group by $O=\left\langle a, b \mid a^{2}=b^{3}=(a b)^{4}=1\right\rangle$, the octahedral group.

Next, we use $\Sigma$ to denote the quotient space of $\mathbb{S}^{2}$ by $O$, and we will find a fundamental region for $\Sigma$ on $\mathbb{S}^{2}$. We first claim that $\triangle 125$ will tile the whole octahedron $\mathbb{S}^{2}$ by the action of $O$. Observe that the action by $a$ sends $\triangle 125$ to $\triangle 216$. Further, $b^{2}(a b) b^{-2}=(1,4,3,2)$ is a $90^{\circ}$ rotation about the axis passing through vertices 5 and 6 , which shows our claim.

Note that the number of fundamental regions for $\Sigma$ on $\mathbb{S}^{2}$ must be 24 as the number is the order of the octahedral group $O=\mathbb{S}_{4}$. Since the $\mathbb{S}^{2}$ currently has eight faces, we will have to triangulate them further. Our approach is that we will add one more vertex on the barycenter on each triangle. For instance, one of the triangulations on $\triangle 125$ is shown in the Figure 2.

We now show that $\triangle 12 y$ becomes a fundamental region for $\Sigma$. Since a rotational axis of $b$ passes the vertex $y$, the barycenter of $\triangle 125$, one can see that $b$ permutes those three triangles $\triangle 12 y, \triangle 51 y$ and $\triangle 25 y$. In the meantime, edges $\overline{1, x_{1}}$ and $\overline{2, x_{1}}$ are identified 


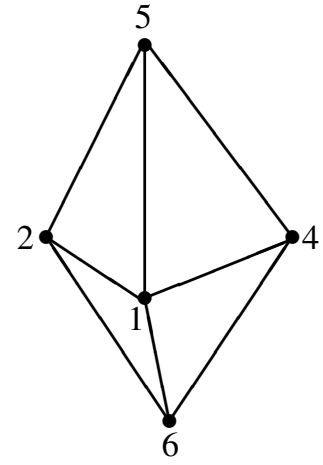

(a) Front of $\mathbb{S}^{2}$.

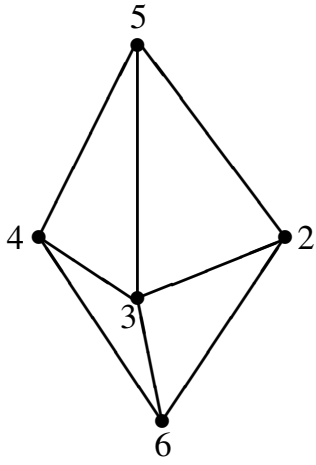

(b) Back of $\mathbb{S}^{2}$.

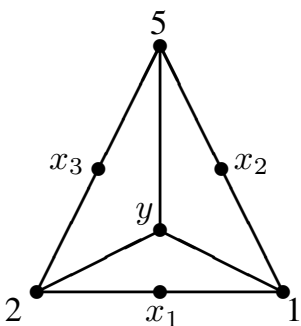

(c) Triangle 125 .

Figure 2: $\mathbb{S}^{2}$ as an octahedron.

by $a$. Likewise, edges $\overline{1, y}$ and $\overline{2, y}$ are identified by $b$. Points $1, x_{1}$ and $y$ will be cone points under the action. Each has an order 4, 2 and 3 respectively. Consequently, we obtain $\Sigma=\mathbb{S}^{2} / O=\Sigma(2,3,4)$.

In order to obtain $O^{h}$, we consider an action $i=(1,3)(2,4)(5,6)$ which is the antipodal map on $\mathbb{S}^{2}$. Notice that the antipodal map commutes with the elements in $O$, hence it induces the reflection map on $\mathbb{S}^{2} / O=\Sigma(2,3,4)$. Now, we choose a triangle whose vertices are $1, x_{1}$ and $y$. Apply $(a b)^{2} b(a b) i$ on the triangle gives us the triangle with vertices $2, x_{1}$ and $y$. Notice that segments $1 x_{1}$ and $2 x_{1} ; 1 y$ and $2 y$ have been identified under $O$-action and the segment $x_{1} y$ has been fixed under the map $(a b)^{2} b(a b) i$. This argument shows that $\triangle 1 x_{1} y$ is a fundamental region for $O \times\langle i\rangle$-action on $\mathbb{S}^{2}$. The vertices of $\triangle 1 x_{1} y$ become corner reflectors, and the edges minus the vertices become the reflector lines. As a result, $\mathbb{S}^{2} /[O \times\langle i\rangle]=O^{h}$, where $O \times\langle i\rangle=\mathbb{S}_{4} \times \mathbb{Z}_{2}=\pi_{1}\left(O^{h}\right)$.

We remark that $\mathbb{S}^{2} /\langle i\rangle=\mathbb{P}^{2}$ is the projective plane. Since the antipodal map $i$ commutes with $O$, the octahedral action on $\mathbb{S}^{2}$ induces the action generated by $\bar{a}$ and $\bar{b}$ on $\mathbb{P}^{2}$, which is isomorphic to the octahedral group $O$. As a result, we also obtain $\mathbb{P}^{2} /\langle\bar{a}, \bar{b}\rangle=O^{h}$. We will now describe the octahedral action $O$ on $\mathbb{P}^{2}$.

The left diagram in Figure 3 illustrates a fundamental region on $\mathbb{S}^{2}$ used to obtain $\mathbb{P}^{2}$ under the antipodal map $i=(1,3)(2,4)(5,6)$. For any arc $\overline{x, y, z}$, we let $[\overline{x, y, z}]$ be its projection in $\mathbb{P}^{2}$. The arc $\overline{1,2,3}$ (or $\overline{3,4,1}$ etc) on $\mathbb{S}^{2}$ projects to an orientation reversing loop $[\overline{1,2,3}]$ on $\mathbb{P}^{2}$. The generator $\bar{a}$ maps the loop $[\overline{1,2,3}]$ onto $[\overline{2,1,4}]=[\overline{2,1}][\overline{1,4}]=$ $[\overline{2,1}][\overline{3,2}]$, which traces the same loop as $[\overline{1,2,3}]$ starting at a different point. Thus $\bar{a}$ leaves the loop $[1,2,3]$ invariant and restricted to this loop is a rotation. On the other hand, the map $\bar{b}$ maps the loop $[\overline{1,2,3}]$ onto $[\overline{2,5,4}]$ whose image is shown as a bold line in the middle diagram in Figure 3 above. Moreover, $\bar{b}^{2}$ maps the loop $[\overline{1,2,3}]$ onto the loop $[\overline{1,3,5]}$. Thus the $\mathbb{Z}_{3}$-action generated by $\bar{b}$ does not leave the orientation reversing loop $[\overline{1,2,3}]$ on $\mathbb{P}^{2}$ invariant.

However, it is important to emphasize that this does not imply the $\mathbb{Z}_{3}$-action leaves no orientation reversing loops invariant. In fact, we can find another orientation reversing loop on $\mathbb{P}^{2}$ which is left invariant under the map $\bar{b}$. It can be found by looking at the octahedron $\mathbb{S}^{2}$ which double covers $\mathbb{P}^{2}$. Consider the circle on $\mathbb{S}^{2}$ which contains the vertices consisting 
of the midpoints of $\overline{4,5}, \overline{5,3}, \overline{3,2}, \overline{2,6}, \overline{6,1}$ and $\overline{1,4}$. One can check that this circle is left invariant under $b$ and the covering translation $i$, hence it projects to an orientation reversing loop on $\mathbb{P}^{2}$ left invariant under $\bar{b}$. It follows that the entire $\mathbb{S}_{4}$-action on $\mathbb{P}^{2}$ does not leave any orientation reversing loop invariant.
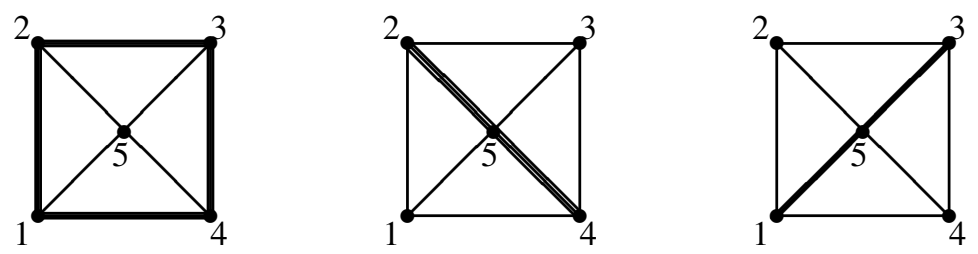

Figure 3: Fundamental region on octahedron.

Lemma 2.1. Let $\mathbb{Z}_{2}$ be a subgroup of $\pi_{1}\left(O^{h}\right)$ such that $\mathbb{P}^{2} \rightarrow O^{h}$ is the covering corresponding to $\mathbb{Z}_{2}$. Then $\mathbb{Z}_{2}=\langle i\rangle$.

Proof. We will show that there is only one element of order two in $O^{h}=\mathbb{S}_{4} \times \mathbb{Z}_{2}$ acting on $\mathbb{S}^{2}$ which is fixed point free and orientation reversing, and that element is $i$. Since the elements in $\mathbb{S}_{4}$ and $\mathbb{Z}_{2}$ commute, we will first look at all elements of order two in $\mathbb{S}_{4}$. In this group, there are nine such elements. Six of them are a rotation of $180^{\circ}$ where their rotational axes are on midpoints of edges. For example, one rotational axis passes the midpoint of $\overline{1,4}$ and $\overline{2,3}$. Another one passes the midpoint of $\overline{2,5}$ and $\overline{4,6}$. Notice that all six types of these rotations are conjugate in $\mathbb{S}_{4}$. Moreover, there are three types of $90^{\circ}$ rotations, call them $r_{1}, r_{2}$ and $r_{3}$, where $r_{1}=(1,2,3,4), r_{2}=(1,6,3,5)$ and $r_{3}=(2,5,4,6)$ respectively. Clearly, they generate three kinds of $180^{\circ}$ rotations which are conjugate in $\mathbb{S}_{4}$. As a result, $\mathbb{S}_{4}$ has two conjugacy classes of order two elements, and we will choose $a$ and $(a b)^{2}$ from the group to represent each class. There is an easy way to verify if two elements in $\mathbb{S}_{n}$ are conjugate for $n \in \mathbb{N}$ by checking their cycle types. (See [2, Chapter 4].) Now, we compose them with the antipodal map $i$ to obtain $a i=(1,4)(2,3)$ and $(a b)^{2} i=(1,3)$. Since both maps have a fixed point, if $\mathbb{P}^{2} \rightarrow O^{h}$ is the covering corresponding to any $\mathbb{Z}_{2}$, then $\mathbb{Z}_{2}=\langle i\rangle$.

Proposition 2.2. Let $\varphi: G \rightarrow \operatorname{Homeo}\left(\mathbb{P}^{2}\right)$ be a finite action such that $\mathbb{P}^{2} / \varphi$ is homeomorphic to $O^{h}$. Then $G \simeq \mathbb{S}_{4}$ and $\varphi$ is conjugate to the standard action $\mathbb{S}_{4}=\langle\bar{a}, \bar{b}\rangle$. Moreover, no orientation reversing loop is left invariant by the $G$-action.

Proof. Let $\nu: \mathbb{P}^{2} \rightarrow \mathbb{P}^{2} /\langle\bar{a}, \bar{b}\rangle$ and $\nu_{\varphi}: \mathbb{P}^{2} \rightarrow \mathbb{P}^{2} / \varphi$ be the orbifold covering maps. By assumption there exists a homeomorphism $h: \mathbb{P}^{2} /\langle\bar{a}, \bar{b}\rangle \rightarrow \mathbb{P}^{2} / \varphi$. By Lemma 2.1 , the $\mathbb{Z}_{2}$ subgroup of $\pi_{1}\left(\mathbb{P}^{2} / \varphi\right)$ giving rise to a covering $\mathbb{P}^{2} \rightarrow \mathbb{P}^{2} / \varphi$ is unique. Hence $h$ lifts to a homeomorphism $\tilde{h}: \mathbb{P}^{2} \rightarrow \mathbb{P}^{2}$ and we obtain the following commutative diagram:

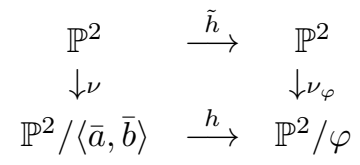

This implies that $G \simeq \mathbb{S}_{4}$ and $\tilde{h}$ conjugates $\varphi$ to the standard action $\langle\bar{a}, \bar{b}\rangle$. 


\section{Chiral dodecahedral/icosahedral symmetry $I$ and achiral dodeca- hedral/icosahedral symmetry $I^{h}$}

We describe the groups $I=\mathbb{A}_{5}$ and $I^{h}=I \times \mathbb{Z}_{2}=\mathbb{A}_{5} \times \mathbb{Z}_{2}$ acting on the 2-sphere $\mathbb{S}^{2}$, and show how $\mathbb{A}_{5}$ acts on $\mathbb{P}^{2}$.

We view $\mathbb{S}^{2}$ as a dodecahedron consisting of 12 pentagons as shown in the first two figures from the left in Figure 4. We also consider two elements $a$ and $b$ in $\mathbb{S}_{20}$ where $a=(1,2)(3,7)(4,13)(5,8)(6,14)(9,12)(10,19)(11,20)(15,18)(16,17)$ and the element $b=(2,5,7)(3,6,13)(4,12,8)(9,11,19)(10,18,14)(15,17,20)$. The two elements act on the dodecahedron $\mathbb{S}^{2}$, and we can see that $a$ is a $180^{\circ}$ rotation about the axis passing through the midpoint of edges $\overline{1,2}$ and $\overline{16,17}$. On the other hand, $b$ is a $120^{\circ}$ rotation about the axis passing through the vertices 1 and 16 . Moreover, $a b^{-1}$ is a $72^{\circ}$ rotation about the axis passing through the barycenter of the pentagon whose vertices are $1,2,3,4,5$ and $16,17,18,19,20$ respectively since $a b^{-1}=(1,2,3,4,5)(6,7,8,9,10)(11,12,13,14,15)$ $(16,17,18,19,20)$. Consequently, $a$ and $b$ generate a group isomorphic to $I=\mathbb{A}_{5}$ written by $I=\left\langle a, b \mid a^{2}=b^{3}=\left(a b^{-1}\right)^{5}=1\right\rangle$.

We use $\Sigma$ to denote the quotient space of $\mathbb{S}^{2}$ by $I$, and we will look for a fundamental region for $\Sigma$ on $\mathbb{S}^{2}$. We will first observe that one of the pentagons consists of vertices 1,2 , 3,4 and 5 tiles the remaining pentagons on $\mathbb{S}^{2}$. This pentagon is sent to the pentagon with the vertices $1,5,6,12,7$ by $b$. Then, $a b^{-1}$ permutes the remaining pentagons in the front of $\mathbb{S}^{2}$. On the other hand, $\left(a b^{-1}\right)^{2}$ sends the vertices $1,5,6,12,7$ to the vertices $4,3,9,15$, 10 . Then, the map $a$ sends them to the vertices $13,7,12,18,19$ on the back of $\mathbb{S}^{2}$. At this stage, one can see that $a b^{-1}$ permutes all pentagons on the back of $\mathbb{S}^{2}$ except the one on the center whose vertices are $16,17,18,19,20$. However, it can be obtained by applying the map $b^{-1}\left(a b^{-1}\right)^{2}$ on the vertices $4,3,9,15,10$.

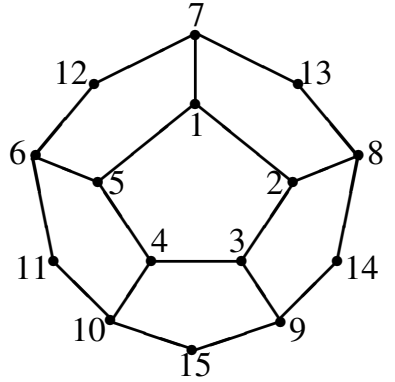

(a) Front of $\mathbb{S}^{2}$.

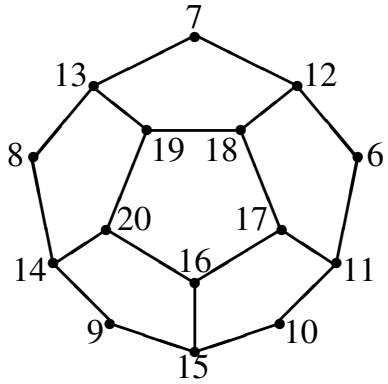

(b) Back of $\mathbb{S}^{2}$

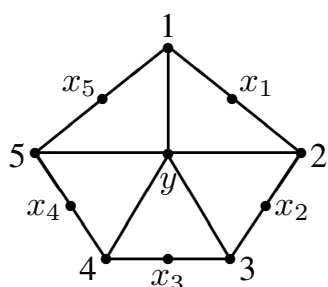

(c) Triangulated face.

Figure 4: $\mathbb{S}^{2}$ as a dodecahedron.

Next, we will add a vertex on the barycenter of the pentagon 1, 2, 3, 4, 5 (see Figure 4), which we denote by $y$. We also add vertices $x_{i}(1 \leq i \leq 5)$ on this pentagon. We can see that $\triangle 12 y$ tiles the remaining triangles on the pentagon $1,2,3,4$ and 5 (see Figure 4) by the map $a b^{-1}$. By the argument above, this proves that $\triangle 12 y$ is a fundamental region for $\Sigma$. Now the edges $\overline{1, x_{1}}$ and $\overline{2, x_{1}}$ are identified by $a \in I$. Likewise, $a b^{-1} \in I$ identifies edges $\overline{1, y}$ and $\overline{2, y}$. The vertices $1, x_{1}$ and $y$ are fixed by the elements $b, a$ and $a b^{-1}$ respectively. Thus, the vertices project to the cone points on $\Sigma$ of orders 3,2 and 5 respectively. Consequently, $\Sigma=\mathbb{S}^{2} / I=\Sigma(2,3,5)$. 
In order to obtain $I^{h}$, we consider an antipodal map on $\mathbb{S}^{2}$ defined by $i=(1,16)(2,17)$ $(3,18)(4,19)(5,20)(6,14)(7,15)(8,11)(9,12)(10,13)$. It is easy to check that the antipodal map commutes with the elements of $I$, hence $I \times \mathbb{Z}_{2}=\langle a, b, i| a^{2}=b^{3}=\left(a b^{-1}\right)^{5}=$ $\left.i^{2}=1,[a, i]=[b, i]=1\right\rangle$. As a result, $i$ induces the reflection map on $\mathbb{S}^{2} / I=\Sigma(2,3,5)$.

We choose a triangle whose vertices are $1, x_{1}$ and $y$ on the fundamental region $\triangle 12 y$. When the map $a\left(a b^{-1}\right)^{3} b^{-1}(a b) i$ is applied on $\triangle 1 x_{1} y$, its image is $\triangle 4 x_{3} y$. Since $\triangle 4 x_{3} y$ is identified to $\triangle 2 x_{1} y$ by $\left(a b^{-1}\right)^{-2} \in I$, this shows the antipodal map $i$ on $\mathbb{S}^{2}$ induces the reflection map on $\Sigma(2,3,5)$ at its equator line. Consequently, $\mathbb{S}^{2} /[I \times\langle i\rangle]=I^{h}$, where $I \times\langle i\rangle=\mathbb{A}_{5} \times \mathbb{Z}_{2}$. The quotient space $\mathbb{S}^{2} /[I \times\langle i\rangle]$ is a mirrored disk where cone points of order 2, 3 and 5 are on the mirror.

Note that $\mathbb{S}^{2} /\langle i\rangle=\mathbb{P}^{2}$ is the projective plane and the map $i$ commutes with $I$. Thus, the icosahedral action on $\mathbb{S}^{2}$ induces the action generated by $\bar{a}$ and $\bar{b}$ on $\mathbb{P}^{2}$, which is isomorphic to the icosahedral group $I$. Hence, we obtain $\mathbb{P}^{2} /\langle\bar{a}, \bar{b}\rangle=I^{h}$.

We will now describe the $I=\mathbb{A}_{5}$-action on $\mathbb{P}^{2}$, and show that it is unique up to conjugation. The front and back of $\mathbb{S}^{2}$ in Figure 4 describe a fundamental region used to obtain $\mathbb{P}^{2}=\mathbb{S}^{2} /\langle i\rangle$ where $i$ is the antipodal map on $\mathbb{S}^{2}$. Note that the boundary of each region in the diagram is left invariant and the interior of each region is exchanged under $i$. The arc $\overline{7,13,8,14,9,15}$ (or $\overline{15,10,11,6,12,7}$ etc) projects to an orientation reversing loop $[\overline{7,13,8,14,9,15}]$ on $\mathbb{P}^{2}$. The map $a b^{-1}$ leaves the outer most loop containing the arc invariant up to the covering translation $i$. Thus, the induced map $\overline{a b^{-1}}$ in $\mathbb{P}^{2}$ leaves this orientation reversing loop invariant. On one hand, $a$ leaves the circle containing vertices $1,2,3$, $9,15,16,17,18,12,7,1$ in $\mathbb{S}^{2}$ invariant which double covers an orientation reversing loop on $\mathbb{P}^{2}$. Note that $\bar{a}$ leaves this orientation reversing loop invariant. However, the orientation reversing loops $[\overline{7,13,8,14,9,15}]$ and $[\overline{3,4,5,6,12,18}]=[\overline{3,4,5,6,9,3}]$ in $\mathbb{P}^{2}$ are exchanged by $\bar{a}$. Finally, $b$ will induce a map $\bar{b}$ on $\mathbb{P}^{2}$. One can see this since three orientation reversing loops in $\mathbb{P}^{2}$, namely $[\overline{7,13,8,14,9,15}],[\overline{2,3,4,10,11,17}]=[\overline{2,3,4,10,8,2}]$ and $[\overline{5,6,12,18,4,5]}=[\overline{5,6,9,3,4,5}]$, are permuted under $\bar{b}$.

Note that although we can find an orientation reversing loop left invariant under $\bar{b}$, no common orientation reversing loop exists which is left invariant by both $\bar{a}$ and $\bar{b}$ since the two maps generate an $\mathbb{A}_{5}$-action on $\mathbb{P}^{2}$.

Lemma 3.1. Let $\mathbb{Z}_{2}$ be a subgroup of $\pi_{1}\left(I^{h}\right)$ such that $\mathbb{P}^{2} \rightarrow I^{h}$ is the covering corresponding to $\mathbb{Z}_{2}$. Then $\mathbb{Z}_{2}=\langle i\rangle$.

Proof. We claim that there is only one element of order two in $I^{h}=\mathbb{A}_{5} \times \mathbb{Z}_{2}$ acting on $\mathbb{S}^{2}$ which is fixed point free and orientation reversing up to a conjugacy. Notice that all elements in $I^{h}$ have the form of $a^{l} b^{m} i^{n}$ for some $l, m, n \in \mathbb{Z}$ where $a, b \in \mathbb{A}_{5}$ and $i \in \mathbb{Z}_{2}$. Since a corresponding covering space must be regular, the group generated by $a^{l} b^{m} i^{n}$ must be a normal subgroup in $I^{h}$. In particular, $a^{l} b^{m}$ generates a normal subgroup of $\mathbb{A}_{5}$ which is impossible unless $l=m=0$. Therefore, a covering space of the orbifold $I^{h}$ corresponding to a $\mathbb{Z}_{2}$ subgroup in $\pi_{1}\left(I^{h}\right)=\mathbb{A}_{5} \times \mathbb{Z}_{2}$ is $\mathbb{S}^{2} /\langle i\rangle=\mathbb{P}^{2}$. Therefore, an $\mathbb{A}_{5}$-action on $\mathbb{P}^{2}$ with quotient type $I^{h}$ is unique up to conjugacy.

Proposition 3.2. Let $\varphi: G \rightarrow \operatorname{Homeo}\left(\mathbb{P}^{2}\right)$ be a finite action such that $\mathbb{P}^{2} / \varphi$ is homeomorphic to $I^{h}$. Then $G \simeq \mathbb{A}_{5}$ and $\varphi$ is conjugate to the standard action $I=\langle\bar{a}, \bar{b}\rangle$. Moreover, no orientation reversing loop is left invariant by the $G$-action.

Proof. The proof is similar to that of Proposition 2.2 and uses the above Lemma 3.1. 


\section{Chiral tetrahedral symmetry $T$ and pyritohedral symmetry $T^{v}$}

We consider the groups $T=\mathbb{A}_{4}$ and $T^{v}=T \times \mathbb{Z}_{2}=\mathbb{A}_{4} \times \mathbb{Z}_{2}$ acting on the 2 -sphere $\mathbb{S}^{2}$ and describe how $T$ acts on the projective plane.
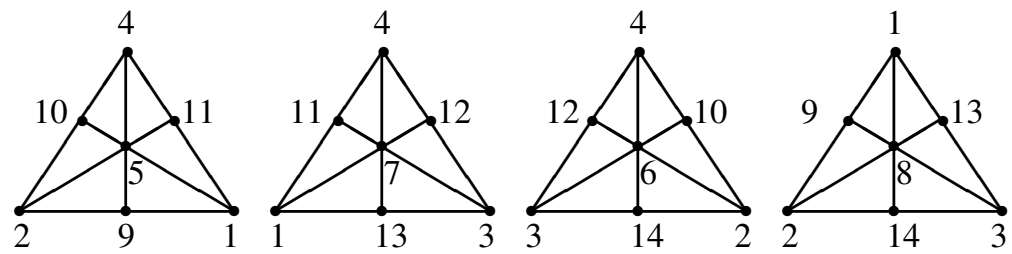

Figure 5: $\mathbb{S}^{2}$ as a tetrahedron.

We view $\mathbb{S}^{2}$ as a tetrahedron which has four faces: $\triangle 124, \triangle 134, \triangle 234$ and $\triangle 123$ (see Figure 5). We add a total of 14 vertices on the faces to triangulate the tetrahedron, and $\triangle 123$ in the Figure 5 illustrates a "bottom" of the tetrahedron.

Consider elements of $\mathbb{S}_{14}$ where $a=(1,2,3)(5,6,7)(9,14,13)(10,12,11)$ and $b=$ $(1,2)(3,4)(5,8)(6,7)(10,13)(11,14)$. These two generators act on the tetrahedron $\mathbb{S}^{2}$. For instance, $a$ is a $120^{\circ}$ rotation about the axis passing throught the vertices 4 and 8 ; and $b$ is a $180^{\circ}$ rotation about the axis passing throught the vertices 9 and 12 . It is easy to see that $a b=(1,3,4)(5,8,6)(9,14,10)(11,13,12)$ is a $120^{\circ}$ rotation about the axis passing through the vertices 2 and 7 . Hence, the two elements $a$ and $b$ generate a group isomorphic to $\mathbb{A}_{4}$, and we call this group by $T=\left\langle a, b \mid a^{3}=b^{2}=(a b)^{3}=1\right\rangle$, which is the tetrahedral group.

Let $\Sigma$ be the quotient space of $\mathbb{S}^{2}$ by the group $T$. We will observe that the face $\triangle 123$ on the "bottom" of this tetrahedron tiles the rest of its faces. To understand this, we look at the map $b$ which sends from $\triangle 123$ to $\triangle 124$. Then, the map $a$ permutes $\triangle 124$ by $120^{\circ}$ each time to tile the whole tetrahedron. However, this argument shows that we may choose $\triangle 128$ for a fundamental region for $\Sigma$ since the map $a$ permutes within the three triangles $\triangle 128, \triangle 238$ and $\triangle 318$ on the "bottom" face $\triangle 123$ of $\mathbb{S}^{2}$.

Notice that $b$, which has the order two, fixes the vertex 9 . Hence, it becomes an exceptional point of order two. Further, $b$ identifies edges $\overline{1,9}$ and $\overline{2,9}$. On the other hand, $a$ fixes the vertex 8 , hence this vertex becomes a cone point of order three. Also, $a$ identifies edges $\overline{1,8}$ and $\overline{2,8}$. Moreover, the map $a b$, which has an order three, fixes the vertex 2 to obtain an additional cone point of order three. Consequently, $\Sigma=\mathbb{S}^{2} / T=\Sigma(2,3,3)$.

Next, we will discuss how to obtain $T^{v}$. An antipodal map defined by $i=(1,6)(2,7)$ $(3,5)(4,8)(9,12)(10,13)(11,14)$ on $\mathbb{S}^{2}$ commutes with the elements in $T$, hence we have $T \times \mathbb{Z}_{2}=\left\langle a, b, i \mid a^{3}=b^{2}=(a b)^{3}=i^{2}=1,[a, i]=[b, i]=1\right\rangle$ and $i$ induces a map on $\mathbb{S}^{2} / T=\Sigma(2,3,3)$. However, it requires some work to analyze what map $i$ induces on the orbifold $\Sigma(2,3,3)$.

First, let $x$ and $y$ be the mid-point of the edge $\overline{1,8}$ and $\overline{2,8}$ respectively. Since the points $x$ and $y$ are identified in $\Sigma(2,3,3)$ by $a \in T$, we may view the union of $\overline{x, 9}$ and $\overline{9, y}$ as the vertical equator line on $\Sigma(2,3,3)$. Notice that the induced map $i$ on $\Sigma(2,3,3)$ fixes all points on the vertical equator line. It can be checked by observing that $a^{-1}(a b)^{-1}\left(a^{2} b\right) i$ fixes the points on the line $\overline{x, 9}$; and $(a b)\left(a^{2} b\right) i$ fixes the points on the line $\overline{9, y}$.

Secondly, we will show that the induced map $i$ on $\Sigma(2,3,3)$ is a reflection on the vertical equator line $\overline{x, 9} \cup \overline{9, y}$. To see this, consider $\triangle 189$ lying on our fundamental 
region $\triangle 128$. Apply the map $a(a b)^{2} i$ on $\triangle 189$ gives us $\triangle 819$ which is a reflection on $\overline{x, 9}$. Likewise, $\triangle 289$ is reflected on $\overline{9, y}$ by the map $a(a b)^{2} b i$ to get $\triangle 829$. As a result, the induced map $i$ on $\Sigma(2,3,3)$ is a reflection at the vertical equator line on the orbifold.

By the argument above, $\mathbb{S}^{2} /[T \times\langle i\rangle]=T^{v}$, where $T \times\langle i\rangle=\mathbb{A}_{4} \times \mathbb{Z}_{2}$ and the quotient space $\mathbb{S}^{2} /[T \times\langle i\rangle]$ is a mirrored disk containing a corner reflector containing one exceptional points of order 2 and 3 on it and one exceptional point of order 3 in its interior.

Recall that the antipodal map $i$ commutes with $T$ on $\mathbb{S}^{2}$, hence $a, b \in T$ induce maps $\bar{a}$ and $\bar{b}$ on $\mathbb{S}^{2} /\langle i\rangle=\mathbb{P}^{2}$. Moreover, $\mathbb{P}^{2} /\langle\bar{a}, \bar{b}\rangle=\mathbb{S}^{2} /[T \times\langle i\rangle]=T^{v}$, where $\langle\bar{a}, \bar{b}\rangle$ is isomorphic to $T$.

We will now describe the $T=\mathbb{A}_{4}$-action on $\mathbb{P}^{2}$ and show that it is unique up to conjugacy.
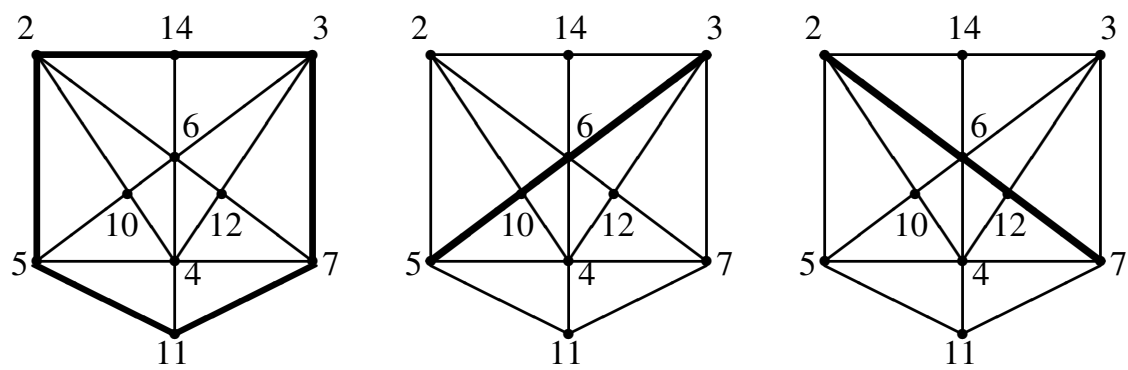

Figure 6: Fundamental region on tetrahedron.

The left diagram in Figure 6 above illustrates a fundamental region on $\mathbb{S}^{2}$ used to obtain $\mathbb{P}^{2}$ under the antipodal map $i$, where $\mathbb{S}^{2}$ is viewed as in Figure 5 and $\mathbb{S}^{2} /\langle i\rangle=\mathbb{P}^{2}$. This can be seen by observing the circle containing vertices, $5,2,14,3,7,11$ is left invariant by $i$, and the vertices $4,10,6,12$ are sent to $8,13,1,9$ respectively. The projective plane is obtained by identifying the opposite side in this polygon.

Recall $a$ and $b$ are generators of the tetrahedral group $T=\mathbb{A}_{4}$ operating on $\mathbb{S}^{2}$. Furthermore $i \notin T=\mathbb{A}_{4}$. Thus $T$ induces an action on $\mathbb{P}^{2}$ and the elements $a, b \in T$ induce maps $\bar{a}, \bar{b}$ on $\mathbb{P}^{2}$. Notice that the generator $\bar{a}$ maps the loop $[\overline{5,2,14,3}]=[\overline{5,2}][\overline{2,14}][\overline{14,3}]$ in $\mathbb{P}^{2}$ onto $[\overline{6,3}][\overline{3,13}][\overline{13,1}]=[\overline{6,3}][\overline{5,10}][\overline{10,6}]=[\overline{6,3,5,10,6}]=[\overline{5,10,6,3}]$, and $\bar{a}^{2}$ maps this loop onto $[2,6,12,7]$. Each image is expressed as a bold line in the Figure 6 above. Thus, the map $\bar{a}$ does not leave this orientation reversing loop invariant in $\mathbb{P}^{2}$. Likewise, $\overline{a b}$ and $\overline{(a b)}^{2}$ map the loop $[\overline{5,2,14,3}]=[\overline{2,14,3,7}]$ onto $[\overline{2,10,4,7}]$ and $[\overline{2,6,12,7}]$ respectively. Furthermore, $\bar{b}$ maps the loop $[\overline{5,2,14,3}]$ onto $[\overline{11,4,6,14}]$. The loop consists of vertices $2,6,12,7,1$ and 9 on the tetrahedron $\mathbb{S}^{2}$ is left invariant by the map $b \in T$ and the covering translation $i$, hence the arc having vertices $2,6,12$ and 7 projects to an orientation reversing loop on $\mathbb{P}^{2}$. There is no orientation reversing loop in $\mathbb{P}^{2}$ which is left invariant by both $\bar{a}$ and $\bar{b}$.

Lemma 4.1. Let $\mathbb{Z}_{2}$ be a subgroup of $\pi_{1}\left(T^{v}\right)$ such that $\mathbb{P}^{2} \rightarrow T^{v}$ is the covering corresponding to $\mathbb{Z}_{2}$. Then $\mathbb{Z}_{2}=\langle i\rangle$.

Proof. We will show that the orbifold $T^{v}$ has only one $\mathbb{P}^{2}$ covering space up to a conjugacy. Notice that $\mathbb{A}_{4}$ has three elements of order two. These elements are $b, a b a^{-1}$ and $a^{2} b a^{-2}$, which are all equivalent. Thus, $\mathbb{A}_{4} \times \mathbb{Z}_{2}$ has two conjugacy classes of order two elements 
which reverse orientation, namely $b i$ and $i$. Since $b i=(1,7)(2,6)(3,8)(4,5)(9,12)$ fixing vertices 10,11, 13 and 14, we have a desired conclusion.

Proposition 4.2. Let $\varphi: G \rightarrow \operatorname{Homeo}\left(\mathbb{P}^{2}\right)$ be a finite action such that $\mathbb{P}^{2} / \varphi=T^{v}$. Then $G \simeq \mathbb{A}_{4}$ and $\varphi$ is conjugate to the standard action generated by $\langle\bar{a}, \bar{b}\rangle$. Moreover, no orientation reversing loop is left invariant by the G-action.

Proof. The proof follows as in Proposition 2.2 and uses Lemma 4.1.

We remark that [4] contains excellent figures to show us how each element in $\mathbb{A}_{4}$ acts on a tetrahedron.

\section{Achiral tetrahedral symmetry $T^{h}$}

In Section 1, we have seen the $O=\mathbb{S}_{4}$-action on $\mathbb{S}^{2}$ where $\mathbb{S}^{2} / O$ is $\Sigma(2,3,4)$, which is an orientable orbifold. In this section, we will investigate another $O=\mathbb{S}_{4}$-action on $\mathbb{S}^{2}$. However, the resulting quotient space $\mathbb{S}^{2} / O=T^{h}$ will be non-orientable this time. More specifically, it will be a mirrored disk which contains two cone points of order three and one cone point of order two on the mirror. Note that we will triangulate $\mathbb{S}^{2}$ as shown in Section 4 which is a tetrahedron.

First, we will begin by providing generators to define a group isomorphic to $\mathbb{S}_{4}$. Consider two elements $a=(1,2)(6,7)(10,11)(13,14)$ and $b=(2,4,3)(5,7,8)(9,11,13)$ $(10,12,14)$ in $\mathbb{S}_{14}$. We can see that $a$ is a reflection on the circle containing vertices 4,5 , $9,8,3$ and 12 in $\mathbb{S}^{2}$. On the other hand, $b$ is a $120^{\circ}$ rotation about the axis passing through vertices 1 and 6 . It is easy to check $a b=(1,2,4,3)(5,6,7,8)(9,10,12,13)(11,14)$. Although $a b$ reverses an orientation, it is called improper rotation. As a result, $\mathbb{S}_{4}=\langle a, b|$ $\left.a^{2}=b^{3}=(a b)^{4}=1\right\rangle$.

Secondly, $\mathbb{A}_{4}$ is an index two subgroup of $\mathbb{S}_{4}$ and the subgroup can be expressed by using the two generators for $\mathbb{S}_{4}$. In order to get a presentation for $\mathbb{A}_{4}$, consider $(a b)^{2}=$ $(1,4)(2,3)(5,7)(6,8)(9,12)(10,13)$ which is a $180^{\circ}$ rotation about the axis passing through vertices 11 and 14 . Then, $b(a b)^{2}=(1,3,4)(5,8,6)(9,14,10)(11,13,12)$ is a $120^{\circ}$ rotation about the axis passing through vertices 2 and 7 . Consequently, we obtain a desired subgroup $\mathbb{A}_{4}=\left\langle b,(a b)^{2} \mid\left[(a b)^{2}\right]^{2}=b^{3}=\left[b(a b)^{2}\right]^{3}=1\right\rangle$.

Thirdly, we will look for a fundamental region for $\mathbb{S}^{2} / \mathbb{A}_{4}$. It is easy to compute that the map $b$ permutes $\triangle 134, \triangle 123$ and $\triangle 142$. Further, $b(a b)^{2}$ maps from $\triangle 123$ to $\triangle 432$. Thus, we will look at $\triangle 134$. However, $\triangle 137$ tiles $\triangle 134$ using the element $b(a b)^{2}$. Then, the vertices 1 and 7 become order 3 cone points since they are fixed by $b$ and $b(a b)^{2}$ respectively. Thus, we may choose $\triangle 137$ for our fundamental region. Notice that the vertex 11 is fixed under $(a b)^{2}$, which becomes the order 2 cone point, and it is identified to the vertex 13 by $b(a b)^{2} \in \mathbb{A}_{4}$. Now, $b(a b)^{2}$ identifies $\overline{1,7}$ and $\overline{3,7} ; b(a b)^{2} b^{-1}$ identifies $\overline{1,13}$ and $\overline{3,13}$. As a result, the quotient space $\mathbb{S}^{2} / \mathbb{A}_{4}$ is indeed $\Sigma(2,3,3)$.

Finally, we will discuss how to obtain the orbifold $T^{h}$. Recall the map $a \in \mathbb{S}_{4}$ reflects on the circle containing vertices $4,5,9,8,3$ and 12 in $\mathbb{S}^{2}$. We compose this map by a covering translation $(a b)^{-1}\left[(a b)^{2} b\right]^{2}(a b)=(1,2,3)(5,6,7)(9,14,13)(10,12,11) \in$ $\mathbb{A}_{4}$, which is a $120^{\circ}$ rotation about the axis passing through vertices 4 and 8 . Then, $(a b)^{-1}\left[(a b)^{2} b\right]^{2}(a b) a$ sends the triangle containing vertices 1,13 and 7 to the triangle containing vertices 3,7 and 13 . Notice that $\overline{1,7}$ and $\overline{3,7}$ are identified in $\Sigma(2,3,3)$. Likewise, $\overline{1,13}$, and $\overline{3,13}$ are identified. Thus, the circle containing vertices $1,7,13$ becomes 
the line of reflection under the map induced by $a$ on $\Sigma(2,3,3)$. Consequently, we obtain $\mathbb{S}^{2} / \mathbb{S}_{4}=T^{h}$.

Unlike the previous orbifolds, $T^{h}$ is not covered by a projective plane. Notice that $\pi_{1}\left(T^{h}\right)=\mathbb{S}_{4}$ contains six elements of order two which are orientation reversing. All of them are a reflection at a plane whose intersection with the tetrahedron is a triangle containing either vertices $2,11,3$; vertices $1,12,2$; vertices $3,10,1$; vertices $4,9,3$; vertices $4,13,2$; or vertices $4,14,1$. Clearly, none of them give a fixed point free action on the tetrahedron $\mathbb{S}^{2}$, and hence this yields the following lemma.

Lemma 5.1. The orbifold $T^{h}$ is not covered by a projective plane.

\section{Cyclic and dihedral actions}

We describe the cyclic and dihedral actions on $\mathbb{S}^{2}$ and the projective plane $\mathbb{P}^{2}$. In describing these actions, it is convenient to use spherical coordinates. Therefore for any point $(x, y, z) \in \mathbb{S}^{2}$, we let $x=\sin \phi \cdot \cos \theta, y=\sin \phi \cdot \sin \theta$ and $z=\cos \phi$.

We begin by defining a rotation of order $m$ on $\mathbb{S}^{2}$ as follows:

$$
r(x, y, z)=\left(\sin \phi \cdot \cos \left(\theta+\frac{2 \pi}{m}\right), \sin \phi \cdot \sin \left(\theta+\frac{2 \pi}{m}\right), \cos \phi\right) .
$$

Note that $r$ fixes only the points $(0,0,1)$ and $(0,0,-1)$.

A spinning map $s$ which rotates through an angle of $\pi$ about the $y$-axis is defined by $s(x, y, z)=(-x, y,-z)$. In terms of the spherical coordinate system, the map is defined by

$$
s(x, y, z)=(\sin (\phi+\pi) \cdot \cos (-\theta), \sin (\phi+\pi) \cdot \sin (-\theta), \cos (\phi+\pi)) .
$$

One can check that $s \circ r \circ s^{-1}=r^{-1}$, and therefore $\langle r, s\rangle$ generates a dihedral group $\operatorname{Dih}\left(\mathbb{Z}_{m}\right)$ acting on $\mathbb{S}^{2}$.

Finally we define the antipodal map $i$ on $\mathbb{S}^{2}$ by $i(x, y, z)=(-x,-y,-z)$. In terms of the spherical coordinate system,

$$
i(x, y, z)=(\sin (\phi+\pi) \cdot \cos \theta, \sin (\phi+\pi) \cdot \sin \theta, \cos (\phi+\pi)) .
$$

We have $\mathbb{S}^{2} /\langle i\rangle=\mathbb{P}^{2}$. Observe that $i \circ s \circ i^{-1}=s$ and $i \circ r \circ i^{-1}=r$. Hence $i$ commutes with $r$ and $s$ which implies the following lemma:

Lemma 6.1. The maps $r$ and $s$ induce homeomorphisms $\bar{r}$ and $\bar{s}$ on $\mathbb{P}^{2}$ respectively.

Let $k(x, y, z)=\left(\sin \phi \cdot \cos \left(\theta+\frac{\pi}{m}\right), \sin \phi \cdot \sin \left(\theta+\frac{\pi}{m}\right), \cos \phi\right)$. A computation shows that $k \circ s \circ k^{-1}=r \circ s, k \circ r \circ k^{-1}=r$ and $k \circ i=i \circ k$. This implies that the induced map $\bar{k}$ on $\mathbb{P}^{2}$ conjugates $\bar{s}$ to $\bar{r} \circ \bar{s}$ and commutes with $\bar{r}$.

Notice that we can express the three maps above in terms of a PL-category. Let $m \in \mathbb{N}$. We assume that vertices from 1 to $2 m$ are located on the equator line of $\mathbb{S}^{2}$. The vertices $2 m+1$ and $2 m+2$ are on the poles. As a result, we obtain $4 m$ many faces (triangles) from these vertices on $\mathbb{S}^{2}$.

If $m>1$ is odd, then the rotation $r$ is expressed by $r=(1,3, \ldots, 2 m-1)(2,4, \ldots, 2 m)$ whose order is $m$. On one hand, if $m$ is even, then $r=(1,2,3, \ldots, 2 m)$ whose order is $2 m$. In each case, the vertices $2 m+1$ and $2 m+2$ are fixed under $r$ since they are the north and the south poles. The spinning map for $m>1$ passing through the $y$-axis is defined by $s=(2,2 m)(3,2 m-1) \cdots(m, m+2)(2 m+1,2 m+2)$. The 
vertices 1 and $m+1$ are fixed under $s$. The antipodal map for $m>1$ is defined by $i=(1, m+1)(2, m+2) \cdots(m, 2 m)(2 m+1,2 m+2)$.

For the case when $r$ has order two, we place vertices 1 to 4 on the equator of $\mathbb{S}^{2}$ and vertices 5 and 6 are on the poles (see the Figure 2 in Section 2). Then $r=(1,3)(2,4)$, $s=(2,4)(5,6)$, and $i=(1,3)(2,4)(5,6)$. Define a map $j=(1,6,3,5)$. A computation shows that $j \circ s \circ j^{-1}=r, j \circ r \circ j^{-1}=s$ and $j \circ i \circ j^{-1}=i$. Therefore $\bar{s}$ is conjugate to $\bar{r}$ on $\mathbb{P}^{2}$. Summarizing we have the following lemma:

Lemma 6.2. There exists a homeomorphism $\bar{k}$ on $\mathbb{P}^{2}$ which conjugates $\bar{s}$ to $\bar{r} \circ \bar{s}$ and commutes with $\bar{r}$. When $\bar{r}$ has order two, there exists a homeomorphism $\bar{j}$ on $\mathbb{P}^{2}$ which conjugates $\bar{r}$ to $\bar{s}$ and $\bar{s}$ to $\bar{r}$.

\subsection{Quotient types $\Sigma(0, m, m), \Sigma(2,2, m), D_{m}^{\nu}$ and $D_{m}^{h}$}

The space $\Sigma(0, m, m)$ is an orbifold whose underlying space is a 2 -sphere with two cone points each of order $m$. Similarly $\Sigma(2,2, m)$ is an orbifold whose underlying space is a 2 -sphere with three cone points, two of order 2 and one of order $m$. The orbifold $D_{m}^{\nu}$ is a mirrored disk containing a cone point of order $m$ and 2 on the mirror and its interior respectively. The orbifold $D_{m}^{h}$ is a mirrored disk with three cone points on the mirror, one of order $m$ and two of order 2 .

Observe that we obtain $\mathbb{S}^{2} /\langle r\rangle=\Sigma(0, m, m)$, which double covers $\mathbb{S}^{2} /\langle r, s\rangle=$ $\Sigma(2,2, m)$. Since $i$ commutes with $r$ and $s$, we have $\operatorname{Dih}\left(\mathbb{Z}_{m}\right) \times \mathbb{Z}_{2}=\left[\langle r\rangle \circ_{-1}\langle s\rangle\right] \times\langle i\rangle$ acting on $\mathbb{S}^{2}$. Now $r$ and $s$ acting on $\mathbb{S}^{2}$ induce a $\operatorname{Dih}\left(\mathbb{Z}_{m}\right)$-action on $\mathbb{S}^{2} /\langle i\rangle=\mathbb{P}^{2}$. Furthermore, $i$ operating on $\mathbb{S}^{2}$ induces an orientation reversing involution $\bar{i}$ on $\Sigma(2,2, m)$, and we have $\Sigma(2,2, m) /\langle\bar{i}\rangle=\mathbb{P}^{2} /\langle\bar{r}, \bar{s}\rangle=\mathbb{S}^{2} /\left(\operatorname{Dih}\left(\mathbb{Z}_{m}\right) \times \mathbb{Z}_{2}\right)$. Thus the fundamental group of the quotient space $\mathbb{P}^{2} /\langle\bar{r}, \bar{s}\rangle$ is $\operatorname{Dih}\left(\mathbb{Z}_{m}\right) \times \mathbb{Z}_{2}=\left[\langle r\rangle \circ_{-1}\langle s\rangle\right] \times\langle i\rangle$.

Let $p: \mathbb{S}^{2} \rightarrow \mathbb{S}^{2} /\langle r, s\rangle=\Sigma(2,2, m)$ be the orbifold covering map and note that $p(0,0,1)=p(0,0,-1)$ is the cone point of order $m$. Since $i(0,0,1)=(0,0,-1)$ and $s(0,0,-1)=(0,0,1)$, it follows that $\bar{i}(p(0,0,1))=p(0,0,1)$, and thus $\bar{i}$ fixes the cone point of order $m$ in $\Sigma(2,2, m)$. Hence $\bar{i}$ is a reflection. If $m$ is odd, $r^{k}(0,1,0) \neq(0,-1,0)$ for any $k$. Thus $p(0,1,0)$ and $p(0,-1,0)$ are the two distinct cone points of order 2 in $\Sigma(2,2, m)$. If $m$ is even, then $p(0,1,0)=p(0,-1,0)$ is a cone point of order 2 since $r^{\frac{m}{2}}(0,1,0)=(0,-1,0)$. We will consider the cases $m$ odd and $m$ even separately.

Suppose $m$ is odd. Then since $i(0,1,0)=(0,-1,0)$, it follows that $\bar{i}(p(0,1,0))=$ $p(0,-1,0)$ and thus $\bar{i}$ exchanges the two cone points of order 2 . Since $\bar{i}$ fixes the cone point of order $m$, it follows that $\mathbb{P}^{2} /\langle\bar{r}, \bar{s}\rangle=D_{m}^{\nu}$. The order two elements in $\operatorname{Dih}\left(\mathbb{Z}_{m}\right) \times \mathbb{Z}_{2}$ are: $i, s, r^{j}$ si. One can check that

$$
r^{j} \operatorname{si}(x, y, z)=\left(\sin \phi \cdot \cos \left(-\theta+\frac{2 \pi j}{m}\right), \sin \phi \cdot \sin \left(-\theta+\frac{2 \pi j}{m}\right), \cos \phi\right) .
$$

By choosing $\phi=0$ or $\pi$, the map fixes the points $(0,0, \pm 1)$ on $\mathbb{S}^{2}$. Note that $\Sigma(0,2,2)=$ $\mathbb{S}^{2} /\langle s\rangle$ is not a regular covering space of $D_{m}^{\nu}$ since $\langle s\rangle$ is not a normal subgroup of $\pi_{1}\left(D_{m}^{\nu}\right)=\operatorname{Dih}\left(\mathbb{Z}_{m}\right) \times \mathbb{Z}_{2}$. Thus $i$ is the only orientation reversing element which is fixed-point free. This implies that when $m$ is odd, $\pi_{1}\left(D_{m}^{\nu}\right)$ has a unique normal $\mathbb{Z}_{2}$ subgroup generated by a fixed-point free orientation reversing element, and the covering of $D_{m}^{\nu}$ corresponding to this subgroup is $\mathbb{P}^{2}$.

Next we suppose $m$ is even and show how to obtain $D_{m}^{\nu}$. Write $m=2 n$ and observe that the rotation $r$ of order $2 n$ on $\mathbb{S}^{2}$ is defined as follows:

$$
r(x, y, z)=\left(\sin \phi \cdot \cos \left(\theta+\frac{\pi}{n}\right), \sin \phi \cdot \sin \left(\theta+\frac{\pi}{n}\right), \cos \phi\right) .
$$


Note that $r$ fixes only the points $(0,0, \pm 1)$, and since $r^{n} s(1,0,0)=(1,0,0)$ it follows that $p(1,0,0)$ is one of the cone points of order 2 in $\Sigma(2,2,2 n)$.

Consider the point $\left(\sin \left(\frac{\pi}{2}\right) \cdot \cos \left(\frac{\pi}{2 n}\right), \sin \left(\frac{\pi}{2}\right) \cdot \sin \left(\frac{\pi}{2 n}\right), \cos \left(\frac{\pi}{2}\right)\right)$. We see that $r^{n+1} s$ fixes $\left(\sin \left(\frac{\pi}{2}\right) \cdot \cos \left(\frac{\pi}{2 n}\right), \sin \left(\frac{\pi}{2}\right) \cdot \sin \left(\frac{\pi}{2 n}\right), \cos \left(\frac{\pi}{2}\right)\right)$, and so it follows that the point $p\left(\left(\sin \left(\frac{\pi}{2}\right) \cdot \cos \left(\frac{\pi}{2 n}\right), \sin \left(\frac{\pi}{2}\right) \cdot \sin \left(\frac{\pi}{2 n}\right), \cos \left(\frac{\pi}{2}\right)\right)\right)$ is the other cone point of order 2 in $\Sigma(2,2, m)$.

Define a reflection $l: \mathbb{S}^{2} \rightarrow \mathbb{S}^{2}$ by

$$
l(x, y, z)=\left(\sin (-\phi) \cdot \cos \left(-\theta+\frac{\pi}{2 n}\right), \sin (-\phi) \cdot \sin \left(-\theta+\frac{\pi}{2 n}\right), \cos (-\phi)\right) .
$$

A calculation shows that $l s l^{-1}=r s$ and $l r l^{-1}=r^{-1}$. Thus we have $\operatorname{Dih}\left(\mathbb{Z}_{2 n}\right) \circ \mathbb{Z}_{2}=$ $\left[\langle r\rangle \circ_{-1}\langle s\rangle\right] \circ\langle l\rangle$ acting on $\mathbb{S}^{2}$ and an induced map $\bar{l}$ acting on $\Sigma(2,2, m)=\mathbb{S}^{2} /\langle r, s\rangle$. A further computation shows that $l(1,0,0)=l\left(\sin \left(\frac{\pi}{2}\right) \cdot \cos (0), \sin \left(\frac{\pi}{2}\right) \cdot \sin (0), \cos \left(\frac{\pi}{2}\right)\right)=$ $\left(\sin \left(\frac{-\pi}{2}\right) \cdot \cos \left(\frac{\pi}{2 n}\right), \sin \left(\frac{-\pi}{2}\right) \cdot \sin \left(\frac{\pi}{2 n}\right), \cos \left(\frac{-\pi}{2}\right)\right)$. Applying $r^{n}$ to this element, we see that

$$
\begin{aligned}
& r^{n}\left(\sin \left(\frac{-\pi}{2}\right) \cdot \cos \left(\frac{\pi}{2 n}\right), \sin \left(\frac{-\pi}{2}\right) \cdot \sin \left(\frac{\pi}{2 n}\right), \cos \left(\frac{-\pi}{2}\right)\right)= \\
& \quad\left(\sin \left(\frac{\pi}{2}\right) \cdot \cos \left(\frac{\pi}{2 n}\right), \sin \left(\frac{\pi}{2}\right) \cdot \sin \left(\frac{\pi}{2 n}\right), \cos \left(\frac{\pi}{2}\right)\right) .
\end{aligned}
$$

Hence the induced map $\bar{l}$ exchanges the two cone points of order two. In addition, consider a set $F \subseteq \mathbb{S}^{2}$ defined by

$$
F=\left\{\left(\sin \varphi \cdot \cos \left(\frac{\pi}{4 n}+\pi\right), \sin \varphi \cdot \sin \left(\frac{\pi}{4 n}+\pi\right), \cos \varphi\right) \mid \varphi \in \mathbb{R}\right\}
$$

Notice that

$$
\begin{aligned}
& l\left(\sin \varphi \cdot \cos \left(\frac{\pi}{4 n}+\pi\right), \sin \varphi \cdot \sin \left(\frac{\pi}{4 n}+\pi\right), \cos \varphi\right) \\
& =\left(\sin (-\varphi) \cdot \cos \left(-\frac{\pi}{4 n}-\pi+\frac{\pi}{2 n}\right), \sin (-\varphi) \cdot \sin \left(-\frac{\pi}{4 n}-\pi+\frac{\pi}{2 n}\right), \cos (-\varphi)\right) \\
& =\left(-\sin \varphi \cdot \cos \left(\frac{\pi}{4 n}+\pi\right),-\sin \varphi \cdot \sin \left(\frac{\pi}{4 n}+\pi\right), \cos (\varphi)\right) \\
& \quad=r^{\frac{m}{2}}\left(\sin \varphi \cdot \cos \left(\frac{\pi}{4 n}+\pi\right), \sin \varphi \cdot \sin \left(\frac{\pi}{4 n}+\pi\right), \cos \varphi\right) .
\end{aligned}
$$

Therefore, $p(F)=\operatorname{fix}\{\bar{l}\}$ in $\Sigma(2,2, m)$ where $p$ denotes the covering map. Consequently, $\bar{l}$ is a reflection exchanging the cone points of order 2 . Thus $\Sigma(2,2, m) /\langle\bar{l}\rangle=D_{2 n}^{v}$, and $\pi_{1}\left(D_{2 n}^{v}\right)=\operatorname{Dih}\left(\mathbb{Z}_{2 n}\right) \circ \mathbb{Z}_{2}=\left[\langle r\rangle \circ_{-1}\langle s\rangle\right] \circ\langle l\rangle$ where $l s l^{-1}=r s$ and $l r l^{-1}=r^{-1}$. The elements of order two are: $r^{n}, r^{k} s, r^{k} l$ (for any integer $k=0,1, \ldots, 2 n-1$ ). The only orientation reversing elements of order two are $r^{k} l$, and they all fix the points $(0,0,1)$ and $(0,0,-1)$. Thus there is no orbifold covering $\mathbb{P}^{2} \rightarrow D_{2 n}^{v}$. We summarize the above in the following theorem.

Theorem 6.3. Let $\varphi: G \rightarrow \operatorname{Homeo}\left(\mathbb{P}^{2}\right)$ be a finite action such that $\mathbb{P}^{2} / \varphi=D_{m}^{\nu}$. Then $m$ is odd, $G \simeq \operatorname{Dih}\left(\mathbb{Z}_{m}\right)$ and $\varphi$ is conjugate to the standard action generated by $\langle\bar{r}, \bar{s}\rangle$.

Proof. By the above $m$ is odd. Let $\nu: \mathbb{P}^{2} \rightarrow D_{m}^{\nu}=\mathbb{P}^{2} /\langle\bar{r}, \bar{s}\rangle$ be the covering map corresponding to the standard action. For the action $\varphi: G \rightarrow \operatorname{Homeo}\left(\mathbb{P}^{2}\right)$ with $\mathbb{P}^{2} / \varphi=D_{m}^{\nu}$, let $\mu: \mathbb{P}^{2} \rightarrow \mathbb{P}^{2} / \varphi$ be the covering map and $h: D_{m}^{\nu} \rightarrow \mathbb{P}^{2} / \varphi$ be a homeomorphism. By the above the subgroup $\mu_{*}\left(\pi_{1}\left(\mathbb{P}^{2}\right)\right)$ in $\pi_{1}\left(\mathbb{P}^{2} / \varphi\right)$ is unique. Thus $h$ lifts to a homeomorphism $\tilde{h}$ of $\mathbb{P}^{2}$ such that $h \nu=\mu \tilde{h}$. This implies that the two actions are conjugate by $\tilde{h}$. 
Suppose $m$ is even. Since $r^{\frac{m}{2}}(0,1,0)=(0,-1,0)$ and $i(0,1,0)=(0,-1,0)$, it follows that $\bar{i}$ fixes the cone point $p(0,1,0)$. Since $\bar{i}$ also fixes the cone point of order $m$, we have that $\bar{i}$ is a reflection leaving each cone point fixed and $\mathbb{P}^{2} /\langle\bar{r}, \bar{s}\rangle=D_{m}^{h}$. The order two elements in $\operatorname{Dih}\left(\mathbb{Z}_{m}\right) \times \mathbb{Z}_{2}$ are: $i, s, r^{j} s i$ or $r^{\frac{m}{2}} i$. Since $r^{j} s i(0,0,1)=(0,0,1)$, we only need to consider

$$
r^{\frac{m}{2}} i(x, y, z)=(\sin (\phi+\pi) \cdot \cos (\theta+\pi), \sin (\phi+\pi) \cdot \sin (\theta+\pi), \cos (\phi+\pi)) .
$$

Letting $\phi=\frac{\pi}{2}$ and $\theta=0$, we see that the point $(1,0,0)$ is fixed by $r^{\frac{m}{2}} i$. Thus $D_{m}^{h}$ has a unique $\mathbb{P}^{2}$ covering up to conjugation. This implies that when $m$ is even, $\pi_{1}\left(D_{m}^{h}\right)$ has a unique normal $\mathbb{Z}_{2}$ subgroup generated by a fixed-point free orientation reversing element, and the covering of $D_{m}^{h}$ corresponding to this subgroup is $\mathbb{P}^{2}$.

We now suppose $m$ is odd and show how to obtain $D_{m}^{h}$. Define a reflection $l_{0}: \mathbb{S}^{2} \rightarrow \mathbb{S}^{2}$ by

$$
l_{0}(x, y, z)=(\sin (-\phi) \cdot \cos (-\theta), \sin (-\phi) \cdot \sin (-\theta), \cos (-\phi))=(-x, y, z) .
$$

One can check that $l_{0} s l_{0}^{-1}=s$ and $l_{0} r l_{0}^{-1}=r^{-1}$. Hence $\operatorname{Dih}\left(\mathbb{Z}_{m}\right) \circ \mathbb{Z}_{2}=\left[\langle r\rangle \circ_{-1}\langle s\rangle\right] \circ\left\langle l_{0}\right\rangle$ acting on $\mathbb{S}^{2}$ and an induced map $\bar{l}_{0}$ acting on $\Sigma(2,2, m)=\mathbb{S}^{2} /\langle r, s\rangle$. Clearly $l_{0}$ fixes the points $(0,1,0)$ and $(0,-1,0)$. Recall $p(0,1,0) \neq(0,-1,0)$. Hence the induced map $\bar{l}_{0}$ on $\Sigma(2,2, m)$ is a reflection which fixes each cone point. Thus $\Sigma(2,2, m) /\left\langle\bar{l}_{0}\right\rangle=D_{m}^{h}$ and $\pi_{1}\left(D_{m}^{h}\right)=\operatorname{Dih}\left(\mathbb{Z}_{m}\right) \circ \mathbb{Z}_{2}=\left[\langle r\rangle \circ_{-1}\langle s\rangle\right] \circ\left\langle l_{0}\right\rangle$.

The elements of order two are: $s, r^{k} s, r^{k} l_{0}$ (any integer $k=0,1, \ldots, m-1$ ), and $s l_{0}$. The only orientation reversing elements of order two are $r^{k} l_{0}$ and $s l_{0}$, but they all have fix-points. Thus there is no orbifold covering $\mathbb{P}^{2} \rightarrow D_{m}^{h}$. We summarize the above in the following theorem whose proof is similar to Theorem 6.3.

Theorem 6.4. Let $\varphi: G \rightarrow \operatorname{Homeo}\left(\mathbb{P}^{2}\right)$ be a finite action such that $\mathbb{P}^{2} / \varphi=D_{m}^{h}$. Then $m$ is even, $G \simeq \operatorname{Dih}\left(\mathbb{Z}_{m}\right)$ and $\varphi$ is conjugate to the standard action generated by $\langle\bar{r}, \bar{s}\rangle$.

\subsection{Quotient types $S^{2 m}$ and $Z_{m}^{h}$}

We use $S^{2 m}$ and $Z_{m}^{h}$ to denote a projective plane that has one cone point of order $m$ and a mirrored disk containing an order $m$ cone point in its interior respectively. The orbifold $Z_{0}^{h}$ denotes a mirrored disk without an exceptional point within its interior, and if $m=1$, then $S^{2(1)}=\mathbb{P}^{2}$. Recall $\langle r\rangle \times\langle i\rangle=\mathbb{Z}_{m} \times \mathbb{Z}_{2}$ acts on $\mathbb{S}^{2}$. Hence, the involutions in this group are either $i, r^{\frac{m}{2}}$ or $i r^{\frac{m}{2}}$ for an even number $m$.

If $m$ is even, then $i r^{\frac{m}{2}}(x, y, z)=(\sin \phi \cdot \cos \theta, \sin \phi \cdot \sin \theta,-\cos \phi)$. The fixed point set of this map is the circle at the equator on $\mathbb{S}^{2}$ and occurs when $\phi=\frac{\pi}{2}$. Thus, $\mathbb{S}^{2} /\left\langle i r^{\frac{m}{2}}\right\rangle=\mathbb{S}^{2} / \mathbb{Z}_{2}=Z_{0}^{h}$. Furthermore, $r$ on $\mathbb{S}^{2}$ induces a rotation $\bar{r}$ on $Z_{0}^{h}$ fixing a point not on the mirror, and inducing an action $\widehat{r}$ acts on $\mathbb{P}^{2}=\mathbb{S}^{2} /\langle i\rangle$. In the meantime, $i$ on $\mathbb{S}^{2}$ induces a reflection $\bar{i}$ on $\Sigma(0, m, m)=\mathbb{S}^{2} /\langle r\rangle$ since $r^{\frac{m}{2}}(-1,0,0)=(1,0,0)$ and $i(1,0,0)=(-1,0,0)$. As a result, we obtain $Z_{m}^{h}=Z_{0}^{h} /\langle\bar{r}\rangle=\mathbb{P}^{2} /\langle\widehat{r}\rangle=\Sigma(0, m, m) /\langle\bar{i}\rangle$ for $m$ is even. Note that $\pi_{1}\left(Z_{m}^{h}\right) \simeq \mathbb{Z}_{m} \times \mathbb{Z}_{2}$ is generated by $r$ and $i$, where $i$ is the only fixed-point free orientation reversing element. This implies that when $m$ is even, $\pi_{1}\left(Z_{m}^{h}\right)$ has a unique normal $\mathbb{Z}_{2}$ subgroup generated by a fixed-point free orientation reversing element, and the covering of $Z_{m}^{h}$ corresponding to this subgroup is $\mathbb{P}^{2}$.

We now show how to obtain $Z_{m}^{h}$ when $m$ is odd. Let $\rho$ be a homeomorphism of $\mathbb{S}^{2}$ defined by $\rho(x, y, z)=(\sin \phi \cdot \cos \theta, \sin \phi \cdot \sin \theta,-\cos \phi)$. A computation shows that 
$\rho$ and $r$ commute. We obtain an orbifold covering map $\mathbb{S}^{2} \rightarrow \Sigma(0, m, m)=\mathbb{S}^{2} /\langle r\rangle$ with $\rho$ inducing a reflection $\bar{\rho}$ on $\Sigma(0, m, m)$. The quotient space $\Sigma(0, m, m) /\langle\bar{\rho}\rangle=Z_{m}^{h}$ and $\pi_{1}\left(Z_{m}^{h}\right) \simeq \mathbb{Z}_{m} \times \mathbb{Z}_{2}$ is generated by $r$ and $\rho$. Since $m$ is odd, the only element of order 2 in $\pi_{1}\left(Z_{m}^{h}\right)$ is $\rho$ which has fixed points. Thus there is no orbifold covering $\mathbb{P}^{2} \rightarrow Z_{m}^{h}$ when $m$ is odd. Consequently, the following theorem is obtained:

Theorem 6.5. Let $\varphi: G \rightarrow \operatorname{Homeo}\left(\mathbb{P}^{2}\right)$ be a finite action. If $\mathbb{P}^{2} / \varphi=Z_{m}^{h}$, then $m$ is even, $G \simeq \mathbb{Z}_{m}$ and $\varphi$ is conjugate to the standard action generated by $\langle\widehat{r}\rangle$.

If $m$ is odd, we again have $r$ inducing a map $\widehat{r}$ on $\mathbb{P}^{2}=\mathbb{S}^{2} /\langle i\rangle$, and one can check that the induced map $\bar{i}$ on $\Sigma(0, m, m)=\mathbb{S}^{2} /\langle r\rangle$ is the antipodal map. Consequently, we obtain $S^{2 m}=\mathbb{P}^{2} /\langle\widehat{r}\rangle=\Sigma(0, m, m) /\langle\bar{i}\rangle$. Furthermore $\pi_{1}\left(S^{2 m}\right) \simeq \mathbb{Z}_{m} \times \mathbb{Z}_{2}$ is generated by $r$ and $i$, where the only order two fixed-point free orientation reversing element is $i$. Hence when $m$ is odd, $\pi_{1}\left(S^{2 m}\right)$ has a unique normal $\mathbb{Z}_{2}$ subgroup generated by a fixed-point free orientation reversing element, and the covering of $S^{2 m}$ corresponding to this subgroup is $\mathbb{P}^{2}$.

To obtain $S^{2 m}$ when $m$ is even, we write $m=2 n$ and define a homeomorphism $h$ of $\mathbb{S}^{2}$ by $h(x, y, z)=\left(\sin (\phi+\pi) \cdot \cos \left(\theta+\frac{\pi}{2 n}\right), \sin (\phi+\pi) \cdot \sin \left(\theta+\frac{\pi}{2 n}\right), \cos (\phi+\pi)\right)$. Observe that $h$ is a composition of the antipodal map and a rotation through $\pi / 2 n$, and $h$ generates a $\mathbb{Z}_{2(2 n)}$-action on $\mathbb{S}^{2}$ and $\mathbb{S}^{2} /\left\langle h^{2}\right\rangle=\Sigma(0,2 n, 2 n)$. It follows that the induced map $\bar{h}$ on $\Sigma(0,2 n, 2 n)$ is the antipodal map and $\Sigma(0,2 n, 2 n) /\langle\bar{h}\rangle=S^{2(2 n)}$. Furthermore $\pi_{1}\left(S^{2(2 n)}\right) \simeq \mathbb{Z}_{2(2 n)}$ is generated by $h$. The only element of order 2 is $h^{2 n}$, and $h^{2 n}(x, y, z)=(\sin \phi \cdot \cos (\theta+\pi), \sin \phi \cdot \sin (\theta+\pi), \cos \phi)$ has fixed-points. Thus there is no orbifold covering $\mathbb{P}^{2} \rightarrow S^{2 m}$ when $m$ is even. Summarizing these results we obtain the following theorem:

Theorem 6.6. Let $\varphi: G \rightarrow \operatorname{Homeo}\left(\mathbb{P}^{2}\right)$ be a finite action. If $\mathbb{P}^{2} / \varphi=S^{2 m}$, then $m$ is odd, $G \simeq \mathbb{Z}_{m}$ and $\varphi$ is conjugate to the standard action generated by $\langle\widehat{r}\rangle$.

\subsection{Nonexistence of quotient type $C_{m, m}^{\nu}$}

The orbifold $C_{m, m}^{\nu}$ is a mirrored disk with two cone points on the mirror of order $m$. We will show that the orbifold $C_{m, m}^{\nu}$ is obtained by some covering translations on $\mathbb{S}^{2}$. Recall the reflection map on the $y z$-plane defined on $\mathbb{R}^{3}$ by $l_{0}(x, y, z)=(-x, y, z)$ and the rotation $r(x, y, z)=\left(\sin \phi \cdot \cos \left(\theta+\frac{2 \pi}{m}\right), \sin \phi \cdot \sin \left(\theta+\frac{2 \pi}{m}\right), \cos \phi\right)$. It is easy to check that $\operatorname{Dih}\left(\mathbb{Z}_{m}\right)=\langle r\rangle \circ_{-1}\left\langle l_{0}\right\rangle$. Then, we obtain $\Sigma(0, m, m)=\mathbb{S}^{2} /\langle r\rangle$ and the reflection on $\mathbb{S}^{2}$ induces a reflection $\overline{l_{0}}$ on $\Sigma(0, m, m)$. As a result, $C_{m, m}^{\nu}=\Sigma(0, m, m) /\left\langle\overline{l_{0}}\right\rangle$ where $\pi_{1}\left(C_{m, m}^{\nu}\right)=\operatorname{Dih}\left(\mathbb{Z}_{m}\right)$. The order two elements in $\pi_{1}\left(C_{m, m}^{\nu}\right)$ are $r^{j} l_{0}$ for $0 \leq j \leq m$, or $r^{\frac{m}{2}}$ for $m$ even. A calculation shows that

$$
r^{j} l_{0}(x, y, z)=\left(\sin (-\phi) \cdot \cos \left(-\theta+\frac{2 \pi}{m}\right), \sin (-\phi) \cdot \sin \left(-\theta+\frac{2 \pi}{m}\right), \cos (-\phi)\right),
$$

which has fixed points at $(0,0, \pm 1) \in \mathbb{S}^{2}$ when $\phi=0$ or $\pi$. Since $l_{0}$ and $r^{\frac{m}{2}}$ when $m$ is even, have fixed points, $C_{m, m}^{\nu}$ is not covered by $\mathbb{P}^{2}$. We therefore have shown the following proposition:

Proposition 6.7. The projective plane does not cover $C_{m, m}^{\nu}$. 


\section{$7 \quad$ Finite group actions on $\mathbb{P}^{2}$ and $\mathbb{P}^{2} \times I$}

In this section, we summarize the above results and classify the finite group actions on $\mathbb{P}^{2}$ and $\mathbb{P}^{2} \times I$.

Theorem 7.1. Let $\varphi: G \rightarrow \operatorname{Homeo}\left(\mathbb{P}^{2}\right)$ be a finite group action on $\mathbb{P}^{2}$. Then $G$ is isomorphic to one of the following groups: $\mathbb{S}_{4}, \mathbb{A}_{5}, \mathbb{A}_{4}, \mathbb{Z}_{m}$ or $\operatorname{Dih}\left(\mathbb{Z}_{m}\right)$. The orbifold quotient $\mathbb{P}^{2} / \varphi$ is an orbifold homeomorphic to one of the following orbifolds: $O^{h}, I^{h}, T^{v}, Z_{m}^{h}$, $S^{2 m}, D_{m}^{v}$ or $D_{m}^{h}$. There is only one equivalence class for each group.

(1) $G \simeq \mathbb{S}_{4}$ if and only if $\mathbb{P}^{2} / \varphi=O^{h}$.

(2) $G \simeq \mathbb{A}_{5}$ if and only if $\mathbb{P}^{2} / \varphi=I^{h}$.

(3) $G \simeq \mathbb{A}_{4}$ if and only if $\mathbb{P}^{2} / \varphi=T^{v}$.

(4) $G \simeq \mathbb{Z}_{m}$ and $m$ is even if and only if $\mathbb{P}^{2} / \varphi=Z_{m}^{h}$.

(5) $G \simeq \mathbb{Z}_{m}$ and $m$ is odd if and only if $\mathbb{P}^{2} / \varphi=S^{2 m}$.

(6) $G \simeq \operatorname{Dih}\left(\mathbb{Z}_{m}\right)$ and $m$ odd if and only if $\mathbb{P}^{2} / \varphi=D_{m}^{v}$.

(7) $G \simeq \operatorname{Dih}\left(\mathbb{Z}_{m}\right)$ and $m$ even if and only if $\mathbb{P}^{2} / \varphi=D_{m}^{h}$

Proof. Let $\varphi: G \rightarrow \operatorname{Homeo}\left(\mathbb{P}^{2}\right)$ be a finite group action. Then $\mathbb{P}^{2} / \varphi$ is a non-orientable 2orbifold with positive euler number $\chi\left(\mathbb{P}^{2} / \varphi\right)$. The non-orientable good orbifolds (orbifolds which have manifolds for their universal covering spaces) with positive euler numbers are the following: $C_{m, m}^{v}, S^{2 m}, Z_{m}^{h}, D_{m}^{h}, D_{m}^{v}, T^{h}, O^{h}, I^{h}$ and $T^{v}$. The result then follows by the above.

Theorem 7.2. Let $\varphi: G \rightarrow \operatorname{Homeo}\left(\mathbb{P}^{2}\right)$ be a finite group action. The action $\varphi(G)$ does not leave any orientation reversing loop in $\mathbb{P}^{2}$ invariant if and only if $G$ is isomorphic to $\mathbb{S}_{4}, \mathbb{A}_{5}$ or $\mathbb{A}_{4}$. Furthermore, $\varphi$ is equivalent to one of these standard actions.

Proof. This follows from Sections 2 through 6.

Theorem 7.3. Let $\varphi: G \rightarrow \operatorname{Homeo}\left(\mathbb{P}^{2} \times I\right)$ be a finite group action. If for every $g \in G$ $\varphi(g)\left(\mathbb{P}^{2} \times\{0\}\right)=\mathbb{P}^{2} \times\{0\}$, then $G$ is isomorphic to one of the following groups: $\mathbb{S}_{4}$, $\mathbb{A}_{5}, \mathbb{A}_{4}, \mathbb{Z}_{m}$ or $\operatorname{Dih}\left(\mathbb{Z}_{m}\right)$. Furthermore, there is only one equivalence class for each group which is represented by one of the standard actions.

Proof. By the comment following Theorem 8.1 in [6], we may conjugate $\varphi(G)$ so that it is a product action. This implies that there is a $G$-action $\varphi_{1}: G \rightarrow \operatorname{Homeo}\left(\mathbb{P}^{2}\right)$ such that for any $g \in G$, we have $\varphi(g)(z, t)=\left(\varphi_{1}(g)(z), t\right)$. By Theorem 7.1 , there exists a homeomorphism $k$ of $\mathbb{P}^{2}$ such that $k \varphi_{1}(G) k^{-1}$ is one of the standard actions (1) through (7) listed there. Conjugating this action further by $k \times$ id proves the result.

Theorem 7.4. Let $\varphi: G \rightarrow \operatorname{Homeo}\left(\mathbb{P}^{2} \times I\right)$ be a finite group action. Then $G$ is isomorphic to one of the following groups: $\mathbb{S}_{4}, \mathbb{S}_{4} \times \mathbb{Z}_{2}, \mathbb{A}_{5}, \mathbb{A}_{5} \times \mathbb{Z}_{2}, \mathbb{A}_{4}, \mathbb{A}_{4} \times \mathbb{Z}_{2}, \mathbb{Z}_{m}, \mathbb{Z}_{m} \times \mathbb{Z}_{2}$, $\operatorname{Dih}\left(\mathbb{Z}_{m}\right)$ or $\operatorname{Dih}\left(\mathbb{Z}_{m}\right) \times \mathbb{Z}_{2}$.

(1) If $G$ is isomorphic to $\mathbb{S}_{4}$, then there are two equivalence classes.

(2) If $G$ is isomorphic to either $\mathbb{S}_{4} \times \mathbb{Z}_{2}, \mathbb{A}_{5}, \mathbb{A}_{5} \times \mathbb{Z}_{2}, \mathbb{A}_{4}, \mathbb{A}_{4} \times \mathbb{Z}_{2}, \mathbb{Z}_{m} \times \mathbb{Z}_{2}$ or $\operatorname{Dih}\left(\mathbb{Z}_{m}\right) \times \mathbb{Z}_{2}$, then there is one equivalence class. 
(3) Suppose $G$ is isomorphic to $\mathbb{Z}_{m}$. If $m$ is odd, then there is one equivalence class. If $m$ is even, then there are two equivalence classes.

(4) Suppose $G \simeq \operatorname{Dih}\left(\mathbb{Z}_{m}\right)$. If $m=2$ or if $m$ is odd, then there are two equivalence classes. If $m>2$ and $m$ is even, there are 3 equivalence classes.

Proof. Again by [6], we may assume $\varphi: G \rightarrow \operatorname{Homeo}\left(\mathbb{P}^{2} \times I\right)$ is a product action. Thus there exists a homomorphism $\varphi_{1}: G \rightarrow \operatorname{Homeo}\left(\mathbb{P}^{2}\right)$ such that for any $g \in G$, $\varphi(g)(z, t)=\left(\varphi_{1}(g)(z), t\right)$ or $\varphi(g)(z, t)=\left(\varphi_{1}(g)(z), 1-t\right)$. There exists a $\mathbb{Z}_{2}$-action on $\mathbb{P}^{2} \times I$ generated by a map $R$ defined by $R(z, t)=(z, 1-t)$.

Suppose first that $\varphi_{1}: G \rightarrow \operatorname{Homeo}\left(\mathbb{P}^{2}\right)$ is not one-to-one. This implies there exists an element $g_{0} \neq 1 \in G$ such that $\varphi_{1}\left(g_{0}\right)(z)=z$ for all $z \in \mathbb{P}^{2}$, and so $\varphi\left(g_{0}\right)(z, t)=(z, 1-t)$ or $\varphi\left(g_{0}\right)=R$. Since $R$ commutes with $\left(\varphi_{1}(g)(z), t\right)$ and $\left(\varphi_{1}(g)(z), 1-t\right)$, it follows that $g_{0}$ commutes with every element of $G$. Let $H=\left\{g \in G \mid \varphi(g)(z, t)=\left(\varphi_{1}(g)(z), t\right)\right\}$. If $\varphi(g)(z, t)=\left(\varphi_{1}(g)(z), 1-t\right)$, then $\varphi\left(g^{2}\right)(z, t)=\left(\varphi_{1}\left(g^{2}\right)(z), t\right)$ showing $g^{2} \in H$. It follows that $H$ is an index two normal subgroup of $G$, and $G=H \times \mathbb{Z}_{2}$ where $\mathbb{Z}_{2}=\left\langle g_{0}\right\rangle$. Furthermore $\left.\varphi_{1}\right|_{H}: H \rightarrow \operatorname{Homeo}\left(\mathbb{P}^{2}\right)$ is one-to-one. By Theorem 7.1, $H$ is isomorphic to $\mathbb{S}_{4}, \mathbb{A}_{5}, \mathbb{A}_{4}, \mathbb{Z}_{m}$ or $\operatorname{Dih}\left(\mathbb{Z}_{m}\right)$ and conjugate to one of the standard actions. As in Theorem 7.3, we may conjugate $\left.\varphi\right|_{H}: H \rightarrow \operatorname{Homeo}\left(\mathbb{P}^{2} \times I\right)$ by a homeomorphism $k \times$ id to a standard action, proving the result in this case.

Suppose $\varphi_{1}: G \rightarrow \operatorname{Homeo}\left(\mathbb{P}^{2}\right)$ is one-to-one, and hence is a $G$-action. Note that in this case $R \notin \varphi(G)$. By Theorem 7.1, $G$ is isomorphic to $\mathbb{S}_{4}, \mathbb{A}_{5}, \mathbb{A}_{4}, \mathbb{Z}_{m}$ or $\operatorname{Dih}\left(\mathbb{Z}_{m}\right)$ and conjugate to one of the standard actions. Thus as above by conjugating $\varphi(G)$, we may assume that $\varphi_{1}$ is one of the standard actions. Suppose $G=\mathbb{S}_{4}$ and $\varphi_{1}(G)=\langle\bar{a}, \bar{b}$ $\left.\bar{a}^{2}=\bar{b}^{3}=(\bar{a} \bar{b})^{4}=1\right\rangle$. Let $A$ and $B$ be actions on $\mathbb{P}^{2} \times I$ defined by $A(z, t)=(\bar{a}(z), t)$ and $B(z, t)=(\bar{b}(z), t)$. If $B \circ R \in \varphi(G)$, then $(B \circ R)^{3}=R \in \varphi(G)$, and this would imply that $\varphi_{1}: G \rightarrow \operatorname{Homeo}\left(\mathbb{P}^{2}\right)$ is not one-to-one. Thus $B \in \varphi(G)$ and we either have $A \in \varphi(G)$ or $A \circ R \in \varphi(G)$. Consequently there are two possibilities $\varphi(G)=$ $\langle A, B\rangle$ or $\varphi(G)=\langle A \circ R, B\rangle$, both isomorphic to $\mathbb{S}_{4}$. They are not conjugate since the quotient space $\left(\mathbb{P}^{2} \times I\right) /\langle A, B\rangle$ has two boundary components while the quotient space $\left(\mathbb{P}^{2} \times I\right) /\langle A \circ R, B\rangle$ has only one boundary component. Suppose $G=\mathbb{A}_{5}$ and let $\varphi_{1}(G)=$ $\left\langle\bar{a}, \bar{b} \mid \bar{a}^{2}=\bar{b}^{3}=\left(\bar{a} \bar{b}^{-1}\right)^{5}=1\right\rangle$. As above we obtain actions $A$ and $B$ on $\mathbb{P}^{2} \times I$ defined by $A(z, t)=(\bar{a}(z), t)$ and $B(z, t)=(\bar{b}(z), t)$. We see as in the previous case that $B \circ R \notin \varphi(G)$. Furthermore since $\left(A \circ R \circ B^{-1}\right)^{5}=R$, it follows that $A \circ R \notin \varphi(G)$. Thus $\varphi(G)=\langle A, B\rangle$ with only one equivalence class. The proof is similar for $\mathbb{A}_{4}$. If $G \simeq \mathbb{Z}_{m}$, then when $m$ is odd the action is conjugate to $(r \times \mathrm{id})$; and when $m$ is even the action is conjugate to either $(r \times \mathrm{id})$ or $(r \times \mathrm{id}) \circ R$. We now suppose $\varphi_{1}(G)=\operatorname{Dih}\left(\mathbb{Z}_{m}\right)=\langle\bar{r}, \bar{s}\rangle$. We first suppose $m$ is even. The possible groups for $\varphi(G)$ are: $H_{1}=\langle(\bar{r} \times \mathrm{id}),(\bar{s} \times \mathrm{id})\rangle$, $H_{2}=\langle(\bar{r} \times \mathrm{id}) \circ R,(\bar{s} \times \mathrm{id})\rangle, H_{3}=\langle(\bar{r} \times \mathrm{id}),(\bar{s} \times \mathrm{id}) \circ R\rangle, H_{4}=\langle(\bar{r} \times \mathrm{id}) \circ R,(\bar{s} \times \mathrm{id}) \circ R\rangle$. Clearly, $H_{1}$ is not conjugate to any of the other groups since no element of $H_{1}$ exchanges the boundary components of $\mathbb{P}^{2} \times I$. The element of order two $(\bar{s} \times$ id $)$ in $H_{2}$ does not exchange boundary components, however every element of order two in $\mathrm{H}_{3}$ exchanges boundary components showing $H_{2}$ is not conjugate to $H_{3}$. Similarly, the element $(\bar{r} \times \mathrm{id})$ of order $m$ in $H_{3}$ cannot be conjugate to $(\bar{r} \times$ id $) \circ R$ in $H_{4}$, showing $H_{3}$ and $H_{4}$ are not conjugate. Notice $H_{4}=\langle(\bar{r} \times \mathrm{id}) \circ R,(\bar{s} \times \mathrm{id}) \circ R\rangle=\langle(\bar{r} \times \mathrm{id}) \circ R,(\bar{r} \bar{s} \times \mathrm{id})\rangle$. Using Lemma 6.2, it follows that $H_{2}$ is conjugate to $H_{4}$, showing there are three equivalence classes when $m>2$. When $m=2$, Lemma 6.2 also shows that $H_{2}$ and $H_{3}$ are conjugate, and so we have only two equivalence classes in this case. When $m$ is odd, the only two possibilities are $\langle(\bar{r} \times \mathrm{id}),(\bar{s} \times \mathrm{id})\rangle$ and $\langle(\bar{r} \times \mathrm{id}),(\bar{s} \times \mathrm{id}) \circ R\rangle$. 


\section{Finite actions on twisted I-bundle over $\mathbb{P}^{2}$}

For $\mathbb{S}^{2} \times I$, define a fixed-point free orientation preserving involution $\alpha: \mathbb{S}^{2} \times I \rightarrow \mathbb{S}^{2} \times I$ by $\alpha(z, t)=(i(z), 1-t)$. The manifold $S^{2} \times I /\langle\alpha\rangle=W$ is a twisted I-bundle over the one-sided projective plane $\mathbb{P}^{2}$. Let $\nu: \mathbb{S}^{2} \times I \rightarrow W$ be the covering map and note that $\nu\left(\mathbb{S}^{2} \times\{1 / 2\}\right)=\mathbb{P}^{2}$ is a one-sided projective plane. The levels of $W$ are $\nu\left(\mathbb{S}^{2} \times\{t\}\right)$, and a homeomorphism $h$ of $W$ is level preserving if $h\left(\nu\left(\mathbb{S}^{2} \times\{t\}\right)\right)=\nu\left(\mathbb{S}^{2} \times\{t\}\right)$. We may view $W$ as the set of equivalence classes $\{[z, t] \mid(z, t)$ is equivalent to $(i(z), 1-t)\}$.

Let Homeo $\left(W, \mathbb{P}^{2}\right)$ be the group of homeomorphisms which leave the projective plane $\mathbb{P}^{2}$ invariant. Denote by $\operatorname{Cent}^{p}(\alpha)$ the subgroup of the centralizer of $\alpha$ which leaves $\mathbb{S}^{2} \times$ $\{1 / 2\}$ invariant and preserves the sides of $\mathbb{S}^{2} \times\{1 / 2\}$. Every homeomorphism which leaves $\mathbb{P}^{2}$ invariant lifts to two homeomorphisms of $\mathbb{S}^{2} \times I$, one of which preserves the sides of $\mathbb{S}^{2} \times\{1 / 2\}$ while the other doesn't. Thus for any homeomorphism $f \in \operatorname{Homeo}\left(W, \mathbb{P}^{2}\right)$ there is a unique lift $\tilde{f} \in \operatorname{Cent}^{p}(\alpha)$, and we obtain an isomorphism $\mathcal{L}: \operatorname{Homeo}\left(W, \mathbb{P}^{2}\right) \rightarrow$ $\operatorname{Cent}^{p}(\alpha)$. Note that since $\left.\nu\right|_{\mathbb{S}^{2} \times\{0\}}: \mathbb{S}^{2} \times\{0\} \rightarrow \partial W$ is a homeomorphism, it follows that $f$ is orientation preserving if and only if $\tilde{f}$ is orientation preserving. We obtain the following proposition.

Proposition 8.1. $\mathcal{L}: \operatorname{Homeo}\left(W, \mathbb{P}^{2}\right) \rightarrow \operatorname{Cent}^{p}(\alpha)$ is an isomorphism.

There exists a map $R: \operatorname{Homeo}\left(W, \mathbb{P}^{2}\right) \rightarrow \operatorname{Homeo}\left(\mathbb{P}^{2}\right)$ defined by restricting any homeomorphism to $\mathbb{P}^{2}$.

Proposition 8.2. Let $\varphi: G \rightarrow \operatorname{Homeo}\left(W, \mathbb{P}^{2}\right)$ be an effective orientation preserving $G$ action. Then the restriction $R \varphi: G \rightarrow \operatorname{Homeo}\left(\mathbb{P}^{2}\right)$ is an effective $G$-action.

Proof. Let $\tilde{\varphi}=\mathcal{L} \circ \varphi: G \rightarrow \operatorname{Cent}^{p}(\alpha)$ be an orientation preserving $G$-action on $\mathbb{S}^{2} \times I$. Suppose there exists an element $g \in G$ such that $R \varphi(g)=\mathrm{id}$, and thus $\left.\tilde{\varphi}(g)\right|_{\mathbb{S}^{2} \times\{1 / 2\}}=\mathrm{id}$ or $i$. Since $\tilde{\varphi}(g)$ does not reverse the sides of $\mathbb{S}^{2} \times\{1 / 2\}$ and $\tilde{\varphi}(g)$ is orientation preserving, we have that $\left.\tilde{\varphi}(g)\right|_{\mathbb{S}^{2} \times\{1 / 2\}}=\mathrm{id}$ implying $\tilde{\varphi}(g)=\mathrm{id}$. This implies that $\varphi(g)=\mathrm{id}$ proving the result.

Remark 8.3. The assumption that the $G$-action in Proposition 8.2 be orientation preserving is necessary. For if we define an involution $\rho$ of $W$ by $\rho[z, t]=[z, 1-t]=[i(z), t]$, then $R \rho=\left.\mathrm{id}\right|_{\mathbb{P}^{2}}$ but $\rho \neq \mathrm{id}$ on $W$.

Proposition 8.4. Let $\varphi_{1}, \varphi_{2}: G \rightarrow \operatorname{Homeo}\left(W, \mathbb{P}^{2}\right)$ be two orientation preserving $G$ actions such that $R \varphi_{1}$ and $R \varphi_{1}$ are effective $G$-actions on $\mathbb{P}^{2}$ with $R \varphi_{1}(G)=R \varphi_{2}(G)$. Then there exists a homeomorphism $k$ of $W$ isotopic to the identity such that $k \varphi_{1}(G) k^{-1}=$ $\varphi_{2}(G)$.

Proof. Let $R \varphi_{1}=\bar{\varphi}_{1}$ and $R \varphi_{2}=\bar{\varphi}_{2}$ be the effective $G$-actions on $\mathbb{P}^{2}$. Replacing $\varphi_{2}$ by $\varphi_{2} \bar{\varphi}_{2}^{-1} \bar{\varphi}_{1}$, we may assume $\bar{\varphi}_{1}=\bar{\varphi}_{2}$. Let $\mathcal{L} \varphi_{1}=\tilde{\varphi}_{1}$ and $\mathcal{L} \varphi_{2}=\tilde{\varphi}_{2}$. Since $\varphi_{1}$ and $\varphi_{2}$ are both orientation preserving, it follows that $\left.\tilde{\varphi}_{1}(g)\right|_{\mathbb{S}^{2} \times\{1 / 2\}}=\left.\tilde{\varphi}_{2}(g)\right|_{\mathbb{S}^{2} \times\{1 / 2\}}$ for any $g \in G$.

Consider $\left.\tilde{\varphi}_{i}(G)\right|_{\mathbb{S}^{2} \times[0,1 / 2]}: \mathbb{S}^{2} \times[0,1 / 2] \rightarrow \mathbb{S}^{2} \times[0,1 / 2]$. Again using [1], both these actions are conjugate to product actions, and hence there exist homeomorphisms $k_{i}$ such that $k_{i} \tilde{\varphi}_{i}(G) k_{i}^{-1}$ is a product action. The conjugating maps $k_{i}$ may be chosen to be the identity on $\mathbb{S}^{2} \times\{1 / 2\}$. Since both actions agree on $\mathbb{S}^{2} \times\{1 / 2\}$, we have $k_{1} \tilde{\varphi}_{1}(G) k_{1}^{-1}=k_{2} \tilde{\varphi}_{2}(G) k_{2}^{-1}$. Letting $h=k_{2}^{-1} k_{1}$, we obtain a homeomorphism 
$h: \mathbb{S}^{2} \times[0,1 / 2] \rightarrow \mathbb{S}^{2} \times[0,1 / 2]$, isotopic to the identity relative to $\mathbb{S}^{2} \times\{1 / 2\}$, such that $h\left(\left.\tilde{\varphi}_{1}(G)\right|_{\mathbb{S}^{2} \times[0,1 / 2]}\right) h^{-1}=\left.\tilde{\varphi}_{1}(G)\right|_{\mathbb{S}^{2} \times[0,1 / 2]}$. Extend $h$ to $\mathbb{S}^{2} \times[1 / 2,1]$ by letting $\left.h\right|_{\mathbb{S}^{2} \times[1 / 2,1]}=\left(\left.\alpha\right|_{\mathbb{S}^{2} \times[0,1 / 2]}\right) \circ\left(\left.h\right|_{\mathbb{S}^{2} \times[0,1 / 2]}\right) \circ\left(\left.\alpha\right|_{\mathbb{S}^{2} \times[1 / 2,1]}\right)$ and note that $h$ and $\alpha$ commute. Let $g \in G$ and $z \in \mathbb{S}^{2} \times[1 / 2,1]$. Now we have

$$
\begin{aligned}
h \tilde{\varphi}_{1}(g) h^{-1}(z)=h \tilde{\varphi}_{1}(g)\left(\left.\alpha \circ h^{-1}\right|_{\mathbb{S}^{2} \times[0,1 / 2]} \circ\right. & \alpha)(z)= \\
& \alpha\left(h \tilde{\varphi}_{1}(g) h^{-1}\right)(\alpha(z))=\alpha \tilde{\varphi}_{2}\left(g^{\prime}\right) \alpha(z)
\end{aligned}
$$

for some $g^{\prime} \in G$. Since $\alpha \tilde{\varphi}_{2}\left(g^{\prime}\right) \alpha(z)=\tilde{\varphi}_{2}\left(g^{\prime}\right)(z)$, we have shown $h \tilde{\varphi}_{1}(G) h^{-1}=\tilde{\varphi}_{2}(G)$. Letting $\mathcal{L}^{-1}(h)=k$, we have $h \varphi_{1}(G) h^{-1}=\varphi_{2}(G)$ proving the result.

Remark 8.5. The assumption in Proposition 8.4 that the actions are both orientation preserving is necessary. For example, let $f: \mathbb{S}^{2} \rightarrow \mathbb{S}^{2}$ be an orientation preserving homeomorphism commuting with $i$ such that $f^{2 n}=$ id. Define two $\mathbb{Z}_{2 n}$-actions on $W$ by $F[z, t]=[f(z), t]$ and $G[z, t]=[i f(z), t]$. Clearly $\left.G\right|_{\mathbb{P}^{2}}=\left.F\right|_{\mathbb{P}^{2}}$ since $i$ projects to the identity on $\mathbb{P}^{2}$, however they are not conjugate as $F$ is orientation preserving, and $G$ is orientation reversing.

Corollary 8.6. Let $\varphi_{1}, \varphi_{2}: G \rightarrow \operatorname{Homeo}\left(W, \mathbb{P}^{2}\right)$ be effective orientation preserving $G$ actions such that $R \varphi_{1}(G)=R \varphi_{2}(G)$. Then there exists a homeomorphism $k$ of $W$ isotopic to the identity such that $k \varphi_{1}(G) k^{-1}=\varphi_{2}(G)$.

Proof. By Proposition $8.2 R \varphi_{1}$ and $R \varphi_{1}$ are effective $G$-actions on $\mathbb{P}^{2}$, and so the result follows by Proposition 8.4 .

Proposition 8.7. Let $\varphi: G \rightarrow \operatorname{Homeo}\left(\mathbb{P}^{2}\right)$ be an effective $G$-action. Then $\varphi$ extends to an effective level preserving orientation preserving $G$-action $\widehat{\varphi}$ on $W$.

Proof. By [7] and [8], there exists an action $\tilde{\varphi}: G \rightarrow$ Cent $_{+}(i)$ where Cent $_{+}(i)$ consists of orientation preserving elements in the centralizer $\operatorname{Cent}(i)$ of $i$ in $\operatorname{Homeo}\left(\mathbb{S}^{2}\right)$. Define an action $\theta: G \rightarrow \operatorname{Cent}^{p}(\alpha)$ by $\theta(g)(x, t)=(\tilde{\varphi}(g)(z), t)$. Then $\mathcal{L}^{-1} \theta: G \rightarrow \operatorname{Homeo}\left(W, \mathbb{P}^{2}\right)$ is the extension.

Let $\mathcal{E}\left(\mathbb{P}^{2}, G\right)$ be the set of equivalence classes of effective $G$-actions on $\mathbb{P}^{2}$, and let $\mathcal{E}_{+}(W, G)$ be the set of equivalence classes of effective orientation preserving $G$-actions on $W$. Denote by $\mathcal{E}_{+}\left(\left(W, \mathbb{P}^{2}\right), G\right)$ the subset of $\mathcal{E}_{+}(W, G)$ which have a representative that leaves a one-sided projective plane invariant.

Proposition 8.8. Let $\varphi: G \rightarrow \operatorname{Homeo}(W)$ be a finite action on $W$. Then there exists a one-sided projective plane $P$ such that $\varphi(g)(P)=P$ for all $g \in G$.

Proof. Let Homeo $\left(\mathbb{S}^{2} \times I, \mathbb{S}^{2} \times\{0\}\right)$ be the group of homeomorphisms which leave $\mathbb{S}^{2} \times$ $\{0\}$ invariant. There exists an injection $\mathcal{L}_{0}: \operatorname{Homeo}(W) \rightarrow \operatorname{Homeo}\left(\mathbb{S}^{2} \times I, \mathbb{S}^{2} \times\{0\}\right) \cap$ Cent $(\alpha)$ defined by lifting any homeomorphism to a homeomorphism of $\mathbb{S}^{2} \times I$ leaving $\mathbb{S}^{2} \times\{0\}$ invariant. Letting $\mathcal{L}_{0} \varphi=\tilde{\varphi}: G \rightarrow \operatorname{Homeo}\left(\mathbb{S}^{2} \times I, \mathbb{S}^{2} \times\{0\}\right) \cap \operatorname{Cent}(\alpha)$, we obtain a $G \times \mathbb{Z}_{2}$ action on $\mathbb{S}^{2} \times I$ where the $\mathbb{Z}_{2}$-action is generated by $\alpha$, which projects to the $\varphi$-action on $W$. This action is equivalent to a product action by [1], and thus there is an $G \times \mathbb{Z}_{2}$-invariant 2 -sphere $S$ in $\operatorname{int}\left(\mathbb{S}^{2} \times I\right)$. Furthermore, $\alpha(S)=S$ and $\nu(S)$ is a $\varphi(G)$-invariant projective plane in $W$.

Corollary 8.9. $\mathcal{E}_{+}\left(\left(W, \mathbb{P}^{2}\right), G\right)=\mathcal{E}_{+}(W, G)$. 
Proposition 8.10. Let $P$ be a one-sided projective plane in $W$. Then there exists a homeomorphism $k$ of $W$, isotopic to the identity, such that $k(P)=\mathbb{P}^{2}$.

Proof. Isotope $P$ to intersect $\mathbb{P}^{2}$ in simple closed curves. We may assume the number of curves in $P \cap \mathbb{P}^{2}$ is minimal. We will show that the number of simple closed curves in $P \cap \mathbb{P}^{2}$ is one. Note first that $P \cap \mathbb{P}^{2} \neq \emptyset$, for otherwise $P \subset W-\mathbb{P}^{2}$ which is isomorphic to $\mathbb{S}^{2} \times[0,1)$, and this is impossible. If the number of intersections of $P \cap \mathbb{P}^{2}$ exceeds one, and hence the number of simple closed curves in $P$ exceeds one, then there is a simple closed curve $\delta \in P \cap \mathbb{P}^{2}$ which bounds a disk $\Delta$ in $P$. We may assume $\Delta$ is innermost in $P$, in the sense that $\operatorname{int}(\Delta) \cap \mathbb{P}^{2}=\emptyset$. Since $\delta$ bounds a disk in $W$, it follows that $\delta$ is an orientation preserving loop in $\mathbb{P}^{2}$, and thus bounds a disk $D$ in $\mathbb{P}^{2}$. Now $D \cup \Delta$ is a separating 2-sphere. If $D \cup \Delta$ bounds a ball, then we may isotope $P$ to eliminate $\delta$.

We therefore assume $D \cup \Delta$ does not bound a ball, and is therefore parallel to the sphere boundary $\partial W=\nu\left(\mathbb{S}^{2} \times\{0\}\right)$. Lift $P$ to an $\alpha$-invariant 2 -sphere $S$ in $\mathbb{S}^{2} \times I$, let $\Delta_{1}$ and $\Delta_{2}$ be the two lifts of $\Delta$ in $S$, and let $D_{1}$ and $D_{2}$ be the two lifts of $D$ in $\mathbb{S}^{2} \times\{1 / 2\}$. Denote $\partial \Delta_{i}$ by $\delta_{i}$. We may assume $D_{1} \cup \Delta_{1} \subset \mathbb{S}^{2} \times[0,1 / 2]$ and $D_{2} \cup \Delta_{2} \subset \mathbb{S}^{2} \times[1 / 2,1]$. Furthermore, there is an $\alpha$-invariant simple closed curve $\gamma \in S \cap \mathbb{S}^{2} \times\{1 / 2\}$, separating $\delta_{1}$ and $\delta_{2}$. Note that $\left(\mathbb{S}^{2} \times\{1 / 2\}-\operatorname{int}\left(D_{1}\right)\right)$ is a disk in $\mathbb{S}^{2} \times\{1 / 2\}$ whose boundary is the boundary of the disk $\Delta_{1}$ in $S$. Now $D_{1} \cup \Delta_{1}$ is parallel to $\mathbb{S}^{2} \times\{0\}$, which implies that $\Delta_{1} \cup\left(\mathbb{S}^{2} \times\{1 / 2\}-\operatorname{int}\left(D_{1}\right)\right)$ bounds a ball in $\mathbb{S}^{2} \times[0,1 / 2]$. Thus we may construct an $\alpha$-equivariant isotopy, relative to $\gamma$, which eliminates the intersections $\delta_{1}$ and $\delta_{2}$. Projecting this isotopy to $W$ eliminates the $\delta$-intersection of $P \cap \mathbb{P}^{2}$.

Thus we have shown that $S \cap \mathbb{S}^{2} \times\{1 / 2\}$ is a single simple closed curve $\gamma$ which projects to a non-contractable simple closed curve $\bar{\gamma}$ in $P \cap \mathbb{P}^{2}$. By an argument similar to the one above, there is an $\alpha$-equivariant isotopy, relative to $\gamma$, which isotopes $S$ to $\mathbb{S}^{2} \times$ $\{1 / 2\}$. Projecting this isotopy to $W$, we obtain an isotopy taking $P$ to $\mathbb{P}^{2}$.

Theorem 8.11. The map $\Gamma: \mathcal{E}\left(\mathbb{P}^{2}, G\right) \rightarrow \mathcal{E}_{+}\left(\left(W, \mathbb{P}^{2}\right), G\right)$ defined by extending $G$-actions from $\mathbb{P}^{2}$ to $W$ is a bijection.

Proof. Let $[\varphi] \in \mathcal{E}\left(\mathbb{P}^{2}, G\right)$. By Proposition $8.7, \varphi$ can be extended to a $G$-action $\widehat{\varphi}$ on $W$. Define $\Gamma([\varphi])=[\widehat{\varphi}]$. Suppose $\psi$ is a $G$-action on $\mathbb{P}^{2}$ such that $[\psi]=[\varphi] \in \mathcal{E}\left(\mathbb{P}^{2}, G\right)$. Then there exists a homeomorphism $h$ of $\mathbb{P}^{2}$ such that $h \varphi(G) h^{-1}=\psi(G)$. Lift $h$ to an orientation preserving homeomorphism $k$ of $\mathbb{S}^{2}$ and note that $i k=k i$. Extend $k$ to a homeomorphism $\widehat{k}$ by $\widehat{k}(x, t)=(k(x), t)$. Letting $\widehat{h}=\mathcal{L}^{-1} \widehat{k}$. we see that $\widehat{h}$ is an extension of $h$. Since $R\left(\widehat{h} \widehat{\varphi}(G) \widehat{h}^{-1}\right)=h \varphi(G) h^{-1}=\psi(G)=R(\widehat{\psi}(G))$, it follows by Proposition 8.4 that $[\widehat{\varphi}]=[\widehat{\psi}]$, and thus $\Gamma$ is well defined.

Let $[\delta] \in \mathcal{E}\left(\left(W, \mathbb{P}^{2}\right), G\right)$. Thus there is a one-sided projective plane $P$ such that $\delta(g)(P)=P$ for all $g \in G$. By Proposition 8.10, there exists a homeomorphism of $W$ taking $P$ to $\mathbb{P}^{2}$. This implies that we may choose a representative $\delta^{\prime}$ in $[\delta]$ such that $\delta^{\prime}(g)\left(\mathbb{P}^{2}\right)=\mathbb{P}^{2}$ for all $g \in G$. By Proposition 8.2, the restriction $R \delta^{\prime}$ is an effective $G$ action on $\mathbb{P}^{2}$ and therefore represents an element in $\mathcal{E}\left(\mathbb{P}^{2}, G\right)$. Let $\Gamma\left(\left[R \delta^{\prime}\right]\right)=\left[\widehat{R \delta^{\prime}}\right]$. Since $R\left(\widehat{R \delta^{\prime}}\right)=R \delta^{\prime}$, it follows by Proposition 8.4 that $\left[\widehat{R \delta^{\prime}}\right]=\left[\delta^{\prime}\right]$, and thus $\Gamma\left(\left[R \delta^{\prime}\right]\right)=\left[\delta^{\prime}\right]$ showing $\Gamma$ is a surjection.

To show $\Gamma$ is one-to-one, suppose that $[\varphi],[\theta] \in \mathcal{E}\left(\mathbb{P}^{2}, G\right)$ are such that their level preserving extensions $[\widehat{\varphi}]=[\widehat{\theta}]$ in $\mathcal{E}_{+}\left(\left(W, \mathbb{P}^{2}\right), G\right)$. Now $W / \varphi$ and $W / \theta$ are homeomorphic twisted I-bundle orbifolds over one of the following 2-orbifolds: $O^{h}, I^{h}, T^{v}, Z_{m}^{h}, S^{2 m}$, $D_{m}^{v}$ or $D_{m}^{h}$. Since by Theorem 7.1, there is only one equivalence class for each action 
on $\mathbb{P}^{2}$ which determines a unique quotient type, it follows that $[\varphi]=[\theta]$ showing $\Gamma$ is one-to-one.

Corollary 8.12. Let $\varphi: G \rightarrow \operatorname{Homeo}(W)$ be a finite orientation preserving $G$-action on $W$. Then $G$ is isomorphic to one of the following groups: $\mathbb{S}_{4}, \mathbb{A}_{5}, \mathbb{A}_{4}, \mathbb{Z}_{m}$ or $\operatorname{Dih}\left(\mathbb{Z}_{m}\right)$. The orbifold quotient for each action is a twisted I-bundle orbifold over the following 2-orbifolds: $O^{h}\left(\right.$ for $\left.\mathbb{S}_{4}\right), I^{h}$ (for $\mathbb{A}_{5}$ ), $T^{v}$ (for $\left.\mathbb{A}_{4}\right), Z_{m}^{h}$ (for $\mathbb{Z}_{m}$ and $m$ even), $S^{2 m}$ (for $\mathbb{Z}_{m}$ and $m$ odd $), D_{m}^{v}$ (for $\operatorname{Dih}\left(\mathbb{Z}_{m}\right)$ and $m$ odd $)$ and $D_{m}^{h}\left(\right.$ for $\operatorname{Dih}\left(\mathbb{Z}_{m}\right)$ and $m$ even $)$. There is one equivalence class for each quotient type.

\section{Orientation reversing finite actions on twisted I-bundle over $\mathbb{P}^{2}$}

Recall that $W=\{[z, t] \mid(z, t)$ is equivalent to $(i(z), 1-t)\}$ with $\mathbb{P}^{2}=\{[z, 1 / 2] \in W\}$, and $\mathcal{L}: \operatorname{Homeo}\left(W, \mathbb{P}^{2}\right) \rightarrow \operatorname{Cent}^{p}(\alpha)$ is an isomorphism. Let $f_{1}$ be a homeomorphism of $\mathbb{P}^{2}$, and let $\tilde{f}_{1}$ be a lift of $f_{1}$ to $\mathbb{S}^{2}$. We remark that $\tilde{f}_{1}$ commutes with $i$. A homeomorphism $f: W \rightarrow W$ is a product homeomorphism if $f[z, t]=\left[\tilde{f}_{1}(z), t\right]$. Note that $\left.f\right|_{\mathbb{P}^{2}}=f_{1}$. Let Homeo $\left(\mathbb{S}^{2} \times I, \mathbb{S}^{2} \times\{1 / 2\}\right)$ be the group of homeomorphisms of $\mathbb{S}^{2} \times I$ which leave $\mathbb{S}^{2} \times\{1 / 2\}$ invariant. Define the map $\tilde{R}: \operatorname{Homeo}\left(\mathbb{S}^{2} \times I, \mathbb{S}^{2} \times\{1 / 2\}\right) \rightarrow \operatorname{Homeo}\left(\mathbb{S}^{2}\right)$ by restricting any homeomorphism to $\mathbb{S}^{2} \times\{1 / 2\}$.

Lemma 9.1. Let $\varphi: G \rightarrow \operatorname{Homeo}\left(W, \mathbb{P}^{2}\right)$ be an effective $G$-action and let $\tilde{\varphi}=\mathcal{L} \varphi: G \rightarrow$ $\operatorname{Cent}^{p}(\alpha) \subset \operatorname{Homeo}\left(\mathbb{S}^{2} \times I, \mathbb{S}^{2} \times\{1 / 2\}\right)$. Then $\tilde{R} \tilde{\varphi}: G \rightarrow \operatorname{Homeo}\left(\mathbb{S}^{2}\right)$ is an effective G-action.

Proof. Suppose there exists an element $g \in G$ such that $\tilde{R} \tilde{\varphi}(g)=\left.i d\right|_{\mathbb{S}^{2} \times\{1 / 2\}}$. Since $\tilde{R} \tilde{\varphi}(g)$ does not reverse the sides of $\mathbb{S}^{2} \times\{1 / 2\}$, it follows that $\tilde{\varphi}(g)=$ id.

Remark 9.2. Note that the involution $\rho$ of $W$ defined by $\rho[z, t]=[i(z), t]=[z, 1-t]$, has the property that $R \rho=\left.i d\right|_{\mathbb{P}^{2}}$, but $\tilde{R} \mathcal{L} \rho(z, t)=(i(z), t)$ and thus does not restrict to the identity on $\mathbb{S}^{2} \times\{1 / 2\}$.

Theorem 9.3. Let $\varphi: G \rightarrow \operatorname{Homeo}(W)$ be an effective $G$-action. Then $\varphi$ is conjugate to a product action on $W$.

Proof. By Propositions 8.8 and 8.10, we may assume $\varphi(g)\left(\mathbb{P}^{2}\right)=\mathbb{P}^{2}$ for every $g \in G$. Let $\tilde{\varphi}=\mathcal{L} \varphi: G \rightarrow \operatorname{Cent}^{p}(\alpha)$. By Lemma 9.1, $\tilde{R} \tilde{\varphi}: G \rightarrow \operatorname{Homeo}\left(\mathbb{S}^{2}\right)$ is an effective $G$-action which commutes with $i$. Define an action $\tilde{\theta}: G \rightarrow \operatorname{Cent}^{p}(\alpha) \subset \operatorname{Homeo}\left(\mathbb{S}^{2} \times I, \mathbb{S}^{2} \times\{1 / 2\}\right)$ by $\tilde{\theta}(g)=\tilde{R} \tilde{\varphi}(g) \times i d$. Thus $\left.\tilde{\theta}(g)\right|_{\mathbb{S}^{2} \times\{1 / 2\}}=\left.\tilde{\varphi}(g)\right|_{\mathbb{S}^{2} \times\{1 / 2\}}$ for any $g \in G$. Projecting this action to $W$, we obtain an effective product action $\theta: G \rightarrow \operatorname{Homeo}\left(W, \mathbb{P}^{2}\right)$. We now use the proof in Proposition 8.4 to construct a homeomorphism $h$ which commutes with $\alpha$ and conjugates $\tilde{\theta}(G)$ to $\tilde{\varphi}(G)$. The homeomorphism $h$ projects to a homeomorphism of $W$ which conjugates $\theta(G)$ to $\varphi(G)$, thus completing the proof.

We will now define the standard actions $\mathbb{S}_{4} \times \mathbb{Z}_{2}, \mathbb{A}_{4} \times \mathbb{Z}_{2}, \mathbb{A}_{4} \times \mathbb{Z}_{2}, \mathbb{Z}_{m} \times \mathbb{Z}_{2}$ or $\operatorname{Dih}\left(\mathbb{Z}_{m}\right) \times \mathbb{Z}_{2}$ on $W$. Consider first the group $\mathbb{S}_{4}=\left\langle a, b \mid a^{2}=b^{3}=(a b)^{4}=1\right\rangle$ acting on $\mathbb{S}^{2}$ commuting with $i$, and its projection $\mathbb{S}_{4}=\left\langle\bar{a}, \bar{b} \mid \bar{a}^{2}=\bar{b}^{3}=(\bar{a} \bar{b})^{4}=1\right\rangle$ to $\mathbb{P}^{2}$. Define the product maps $A, B: W \rightarrow W$ by $A[z, t]=[a(z), t]$ and $B[z, t]=[b(z), t]$. Note that $\langle A, B, \rho\rangle=\mathbb{S}_{4} \times \mathbb{Z}_{2}$. The other standard group actions on $W$ are defined in a similar fashion. 
Theorem 9.4. Let $\varphi: G \rightarrow \operatorname{Homeo}(W)$ be an orientation reversing $G$-action. Then $G$ is isomorphic to one of the following groups: $\mathbb{S}_{4}, \mathbb{Z}_{m}$ with $m$ even, $\operatorname{Dih}\left(\mathbb{Z}_{m}\right), \mathbb{S}_{4} \times \mathbb{Z}_{2}$, $\mathbb{A}_{5} \times \mathbb{Z}_{2}, \mathbb{A}_{4} \times \mathbb{Z}_{2}, \mathbb{Z}_{m} \times \mathbb{Z}_{2}$ or $\operatorname{Dih}\left(\mathbb{Z}_{m}\right) \times \mathbb{Z}_{2}$

(1) If $G$ is either $\mathbb{S}_{4}, \mathbb{S}_{4} \times \mathbb{Z}_{2}, \mathbb{A}_{5} \times \mathbb{Z}_{2}, \mathbb{A}_{4} \times \mathbb{Z}_{2}, \mathbb{Z}_{m} \times \mathbb{Z}_{2}$ with $m$ even or $\operatorname{Dih}\left(\mathbb{Z}_{m}\right)$ with $m$ odd, there is only one equivalence class.

(2) If $G$ is $\mathbb{Z}_{m}$ with $m>2$ even and $m / 2$ odd, then there are two equivalence classes of $\mathbb{Z}_{m}=\mathbb{Z}_{m / 2} \times \mathbb{Z}_{2}$-actions on $W$.

(3) If $G$ is $\mathbb{Z}_{m}$ with either $m / 2$ even or $m=2$, then there is only one equivalence class.

(4) If $G$ is $\operatorname{Dih}\left(\mathbb{Z}_{m}\right)$ with $m>2$ and $m / 2$ even, there are two equivalence classes of $\operatorname{Dih}\left(\mathbb{Z}_{m}\right)$-actions on $W$.

(5) If $G$ is $\operatorname{Dih}\left(\mathbb{Z}_{m}\right)$ with $m>2$ and $m / 2$ odd, there are three equivalence classes of $\operatorname{Dih}\left(\mathbb{Z}_{m}\right)$-actions on $W$.

(6) If $G$ is $\operatorname{Dih}\left(\mathbb{Z}_{m}\right) \times \mathbb{Z}_{2}$ with $m$ even, there is only one equivalence class.

(7) If $G$ is $\operatorname{Dih}\left(\mathbb{Z}_{m}\right) \times \mathbb{Z}_{2}$ with $m$ odd, then $\operatorname{Dih}\left(\mathbb{Z}_{m}\right) \times \mathbb{Z}_{2} \simeq \operatorname{Dih}\left(\mathbb{Z}_{2 m}\right)$ and there are three equivalence classes of $\operatorname{Dih}\left(\mathbb{Z}_{2 m}\right)$-actions on $W$.

Proof. Let $\varphi: G \rightarrow$ Homeo $(W)$ be an effective orientation reversing $G$-action. We may assume by Theorem 9.3, that there exists $G$-actions $\tilde{\varphi}_{1}: G \rightarrow \operatorname{Homeo}\left(\mathbb{S}^{2}\right)$ and $\varphi_{1}: G \rightarrow$ $\operatorname{Homeo}\left(\mathbb{P}^{2}\right)$, such that $\varphi(g)[z, t]=\left[\tilde{\varphi}_{1}(g)(z), t\right]$ and $\tilde{\varphi}_{1}(g)$ is a lift of $\varphi_{1}(g)$. Note that $\tilde{\varphi}_{1}: G \rightarrow \operatorname{Homeo}\left(\mathbb{S}^{2}\right)$ is an effective orientation reversing $G$-action on $\mathbb{S}^{2}$ by Lemma 9.1 .

Suppose that $\varphi_{1}: G \rightarrow \operatorname{Homeo}\left(\mathbb{P}^{2}\right)$ is not an effective $G$-action, and so there exists an element $g_{0} \neq 1 \in G$ such that $\varphi_{1}\left(g_{0}\right)=\mathrm{id}: \mathbb{P}^{2} \rightarrow \mathbb{P}^{2}$. Since $\tilde{\varphi}_{1}\left(g_{0}\right)$ is a lift of $\varphi_{1}\left(g_{0}\right)$ and $\tilde{\varphi}$ is an effective action, we see that $\tilde{\varphi}_{1}\left(g_{0}\right)(z)=i(z)$ and $\varphi\left(g_{0}\right)[z, t]=[i(z), t]=[z, 1-t]$. Thus $\varphi\left(g_{0}\right)=\rho \in \varphi(G)$, and note that $\rho$ commutes with every element in $\varphi(G)$. Let $H=\{g \in G \mid \varphi(g)$ is orientation preserving $\}$, and observe that $G=H \times\left\langle g_{0}\right\rangle$. Since $\left(\left.\varphi\right|_{H}\right): H \rightarrow \operatorname{Homeo}\left(W, \mathbb{P}^{2}\right)$ is an effective orientation preserving action, it follows by Proposition 8.2 that $\left(\left.\varphi_{1}\right|_{H}\right): H \rightarrow \operatorname{Homeo}\left(\mathbb{P}^{2}\right)$ is an effective action. By Theorem 7.1, there exists a homeomorphism $k_{1}: \mathbb{P}^{2} \rightarrow \mathbb{P}^{2}$ such that $k_{1} \varphi_{1}(H) k_{1}^{-1}$ is one of the standard actions $\mathbb{S}_{4}, \mathbb{A}_{5}, \mathbb{A}_{4}, \mathbb{Z}_{m}$ or $\operatorname{Dih}\left(\mathbb{Z}_{m}\right)$ on $\mathbb{P}^{2}$. Lifting $k_{1}$ to a homeomorphism $\tilde{k}_{1}: \mathbb{S}^{2} \rightarrow \mathbb{S}^{2}$, we see that $\tilde{k}_{1} \tilde{\varphi}_{1}(H) \tilde{k}_{1}$ is the same standard action $\mathbb{S}_{4}, \mathbb{A}_{5}, \mathbb{A}_{4}, \mathbb{Z}_{m}$ or $\operatorname{Dih}\left(\mathbb{Z}_{m}\right)$ on $\mathbb{S}^{2}$. Define a homeomorphism $k: W \rightarrow W$ by $k[z, t]=\left[\tilde{k}_{1}(z), t\right]$. Since $\rho[z, t]=[z, 1-t]$, it follows that $k \rho k^{-1}=\rho$. Therefore $k \varphi(G) k^{-1}$ is one of the standard actions: $\mathbb{S}_{4} \times \mathbb{Z}_{2}$, $\mathbb{A}_{5} \times \mathbb{Z}_{2}, \mathbb{A}_{4} \times \mathbb{Z}_{2}, \mathbb{Z}_{m} \times \mathbb{Z}_{2}$ or $\operatorname{Dih}\left(\mathbb{Z}_{m}\right) \times \mathbb{Z}_{2}$, where the $\mathbb{Z}_{2}$ group is generated by $\rho$.

Assume first that $\varphi_{1}: G \rightarrow \operatorname{Homeo}\left(\mathbb{P}^{2}\right)$ is an effective $G$-action on $\mathbb{P}^{2}$. Hence by Theorem 7.1, $\varphi_{1}(G)$ is conjugate to one of the following standard actions on $\mathbb{P}^{2}: \mathbb{S}_{4}, \mathbb{A}_{5}$, $\mathbb{A}_{4}, \mathbb{Z}_{m}$ or $\operatorname{Dih}\left(\mathbb{Z}_{m}\right)$.

Suppose there exists a homeomorphism $k_{1}$ of $\mathbb{P}^{2}$ such that $k_{1} \varphi_{1}(G) k^{-1}=\langle\bar{a}, \bar{b}| \bar{a}^{2}=$ $\left.\bar{b}^{3}=(\bar{a} \bar{b})^{4}=1\right\rangle=\mathbb{S}_{4}$. Let $\tilde{k}_{1}$ be a lift of $k_{1}$ to $\mathbb{S}^{2}$, and note that $\tilde{k}_{1} \tilde{\varphi}_{1}(G) \tilde{k}_{1}^{-1}$ projects to $\langle\bar{a}, \bar{b}\rangle$, and is therefore one of the following groups: $\langle a i, b\rangle,\langle a, b i\rangle$ or $\langle a i, b i\rangle$. The group $\langle a, b\rangle$ is not in the list, since it is orientation preserving on $\mathbb{S}^{2}$. Since $(b i)^{3}=i$, it follows that $\langle a, b i\rangle=\langle a i, b i\rangle=\langle a, b\rangle \times\langle i\rangle=\mathbb{S}_{4} \times \mathbb{Z}_{2}$, and hence must be excluded. Thus, $\tilde{k}_{1} \tilde{\varphi}_{1}(G) \tilde{k}_{1}^{-1}=\langle a i, b\rangle$. Define a homeomorphism $k$ of $W$ by $k[z, t]=\left[\tilde{k}_{1}(z), t\right]$, and note that $k \varphi(G) k^{-1}=\langle A \rho, B\rangle \simeq \mathbb{S}_{4}$.

We now suppose there exists a homeomorphism $k_{1}$ of $\mathbb{P}^{2}$ such that $k_{1} \varphi_{1}(G) k^{-1}=$ $\left\langle\bar{a}, \bar{b} \mid \bar{a}^{2}=\bar{b}^{3}=(\bar{a} \bar{b})^{5}=1\right\rangle=\mathbb{A}_{5}$. As in the previous paragraph, there exists a lift $\tilde{k}_{1}$ 
such that $\tilde{k}_{1} \tilde{\varphi}_{1}(G) \tilde{k}_{1}^{-1}$ is one of the following groups: $\langle a i, b\rangle,\langle a, b i\rangle$ or $\langle a i, b i\rangle$. We see that $(b i)^{3}=i$ and $(a i b)^{5}=i$. This implies $\langle a i, b\rangle=\langle a, b i\rangle=\langle a i, b i\rangle=\mathbb{A}_{5} \times \mathbb{Z}_{2}$, and therefore must be excluded. A similar argument shows that $\varphi_{1}(G)$ cannot be conjugate to $\mathbb{A}_{4}$.

Next, assume that $G$ is isomorphic to $\mathbb{Z}_{m}$, and so we have a homeomorphism $k_{1}$ of $\mathbb{P}^{2}$ such that $k_{1} \varphi_{1}(G) k_{1}^{-1}=\langle\bar{r}\rangle$. For the lifted homeomorphism $\tilde{k}_{1}$ on $\mathbb{S}^{2}$, we have $\tilde{k}_{1} \tilde{\varphi}_{1}(G) \tilde{k}_{1}=\langle r i\rangle$. However if $m$ is odd, then $(r i)^{m}=i$, implying $G$ isomorphic to $\mathbb{Z}_{m} \times \mathbb{Z}_{2}$, which is a contradiction. Thus $m$ is even. Let $k$ be a homeomorphism of $W$ defined by $k[z, t]=\left[\tilde{k}_{1}(z), t\right]$. Observe that $k \varphi(G) k^{-1}=\langle R \rho\rangle$ where $R[z, t]=[r(z), t]$ and $R^{m}=$ id. If $m / 2$ is odd and greater than one, then $\mathbb{Z}_{m}=\mathbb{Z}_{m / 2} \times \mathbb{Z}_{2}=\left\langle R^{2}\right\rangle \times\left\langle R^{m / 2} \rho\right\rangle$, and note that $\rho$ is not an element of this group. Thus when $m / 2$ is odd, there are two non-equivalent $\mathbb{Z}_{m}$-actions on $W$. They are $\left\langle R^{2}\right\rangle \times\left\langle R^{m / 2} \rho\right\rangle$ and $\left\langle R^{2}\right\rangle \times\langle\rho\rangle$, the first in which no element restricts to the identity on $\mathbb{P}^{2}$, and the second that has an element which restricts to the identity on $\mathbb{P}^{2}$. If either $m=2$ or $m / 2$ is even, there is only one equivalence class.

Finally, we assume $G$ is isomorphic to $\operatorname{Dih}\left(\mathbb{Z}_{m}\right)$. Again we have a homeomorphism $k_{1}$ of $\mathbb{P}^{2}$ such that $k_{1} \varphi_{1}(G) k_{1}^{-1}=\langle\bar{r}\rangle \circ_{-1}\langle\bar{s}\rangle$, and its lift $\tilde{k}_{1}$ such that $\tilde{k}_{1} \tilde{\varphi}_{1}(G) \tilde{k}_{1}^{-1}$ is one of the following groups: $\langle r i\rangle_{-1}\langle s\rangle=H_{1},\left\langle r{ } \circ_{-1}\langle s i\rangle=H_{2},\left\langle r i{O_{-}}_{-1}\langle s i\rangle=H_{3}\right.\right.$. Consider first the case when $m$ is odd. Since $(r i)^{m}=i$, we obtain $H_{1}=H_{3}=\operatorname{Dih}\left(\mathbb{Z}_{m}\right) \times \mathbb{Z}_{2}$, and so these cases are excluded. Therefore we only consider $H_{2}$. Likewise $\varphi(G)$ is conjugate to the group $\langle R, S \rho\rangle$ where $S[z, t]=[s(z), t]$, and there is one equivalence class. Suppose that $m$ is even. There exists a homeomorphism $k$ of $\mathbb{S}^{2}$ commuting with $r$ and $i$ such that $k s k^{-1}=r s$ (see Section 6 after Lemma 6.1). Therefore for $r i$ and $s i$ in $H_{3}, k r i k^{-1}=$ ri $\in H_{1}$ and $k s i k^{-1}=r s i \in H_{1}$, showing $H_{3}$ is conjugate to $H_{1}$. If $m=2$, there exists a homeomorphism $j$ of $\mathbb{S}^{2}$ commuting with $i$ such that $j r j^{-1}=s$ and $j s j^{-1}=r$ (see Section 6 before Lemma 6.2). This implies that we may conjugate $\varphi(G)$ to either $\langle R \rho, S\rangle$ or $\langle R, S \rho\rangle$ when $m>2$, or to $\langle R \rho, S\rangle$ when $m=2$. If $m>2$, then any generator of $\mathbb{Z}_{m}$ in $\langle R \rho, S\rangle$ is an odd power of $R \rho$ relatively prime to $m$, and thus orientation reversing. On the other hand, any generator of $\mathbb{Z}_{m}$ in $\langle R, S \rho\rangle$ is orientation preserving. Hence these groups cannot be conjugate. This implies that if $m / 2$ is even, there are two equivalence classes of $\operatorname{Dih}\left(\mathbb{Z}_{m}\right)$-actions.

We note that when $m / 2$ is odd and not equal to one, there are three equivalence classes of $\operatorname{Dih}\left(\mathbb{Z}_{m}\right)$-actions. They are $\langle R \rho\rangle \circ_{-1}\langle S\rangle,\langle R\rangle \circ_{-1}\langle S \rho\rangle$ and $\left\langle R^{2} \rho\right\rangle \circ_{-1}\langle S\rangle$. The last group has an element $\left(R^{2} \rho\right)^{m / 2}=\rho$ restricting to the identity on $\mathbb{P}^{2}$, and the group may be viewed as $\left(\left\langle R^{2}\right\rangle \circ_{-1}\langle S\rangle\right) \times\langle\rho\rangle=\operatorname{Dih}\left(\mathbb{Z}_{m / 2}\right) \times \mathbb{Z}_{2}$. This group was identified in the second paragraph of this proof when we assumed $\varphi_{1}: G \rightarrow \operatorname{Homeo}\left(\mathbb{P}^{2}\right)$ was not an effective $G$-action.

The proof is completed by noting that if $G$ is isomorphic to $\operatorname{Dih}\left(\mathbb{Z}_{m}\right) \times \mathbb{Z}_{2}$ and $m$ is odd, then $\operatorname{Dih}\left(\mathbb{Z}_{m}\right) \times \mathbb{Z}_{2}$ is isomorphic to $\operatorname{Dih}\left(\mathbb{Z}_{2 m}\right)$, and this case has already been dealt with.

\section{References}

[1] H. S. M. Coxeter and W. O. J. Moser, Generators and Relations for Discrete Groups, volume 14 of Ergebnisse der Mathematik und ihrer Grenzgebiete, Springer-Verlag, New York, 4th edition, 1980, doi:10.1007/978-3-662-21943-0.

[2] D. S. Dummit and R. M. Foote, Abstract Algebra, John Wiley \& Sons, Hoboken, New Jersey, 3rd edition, 2004. 
[3] W. D. Dunbar, Geometric orbifolds, Rev. Mat. Univ. Complut. Madrid 1 (1988), 67-99, http: //www.mat.ucm.es/serv/revmat/vol1-123/vol1-123f.html.

[4] J. A. Gallian, Contemporary Abstract Algebra, Houghton Mifflin, 5th edition, 2002.

[5] J. L. Gross and T. W. Tucker, Topological Graph Theory, Dover Publications, Mineola, New York, 2001.

[6] W. H. Meeks, III and P. Scott, Finite group actions on 3-manifolds, Invent. Math. 86 (1986), 287-346, doi:10.1007/bf01389073.

[7] D. Singerman, Automorphisms of compact non-orientable Riemann surfaces, Glasgow Math. J. 12 (1971), 50-59, doi:10.1017/s0017089500001142.

[8] T. W. Tucker, Finite groups acting on surfaces and the genus of a group, J. Comb. Theory Ser. B 34 (1983), 82-98, doi:10.1016/0095-8956(83)90009-6.

[9] B. P. Zimmermann, On finite groups acting on spheres and finite subgroups of orthogonal groups, Sib. Èlektron. Mat. Izv. 9 (2012), 1-12, http://semr.math.nsc.ru/v9/p1-12.pdf. 